\title{
Finite integral transform method for analytical solutions of static problems of cylindrical shell panels
}

\author{
Dongqi An ${ }^{a}$, Dian $\mathrm{Xu}^{\text {a }}$, Zhuofan $\mathrm{Ni}^{\text {a }}$, Yewang Su ${ }^{\text {b,c,a }}$, Bo Wang ${ }^{\text {a }}$, Rui $\mathrm{Li}^{\mathrm{a}}$," \\ ${ }^{\text {a }}$ State Key Laboratory of Structural Analysis for Industrial Equipment, Department of Engineering Mechanics, and International Research Center for Computational \\ Mechanics, Dalian University of Technology, Dalian, 116024, China \\ ${ }^{\mathrm{b}}$ State Key Laboratory of Nonlinear Mechanics, Institute of Mechanics, Chinese Academy of Sciences, Beijing, 100190, China \\ ${ }^{\mathrm{c}}$ School of Engineering Science, University of Chinese Academy of Sciences, Beijing, 100049, China
}

\section{A R T I C L E I N F O}

\section{Keywords:}

Finite integral transform

Analytical solution

Cylindrical shell

Static problem

\begin{abstract}
A B S T R A C T
In this paper, a double finite integral transform method is developed for analytical bending solutions of nonLévy-type cylindrical shell panels without a free edge that were not obtained by classical semi-inverse methods. Three double finite integral transforms are imposed on the governing high-order partial differential equations, which, with some boundary conditions, yields the relationship between the transformed quantities and specific unknowns. Incorporating the inversions into the remaining boundary conditions leads to systems of linear algebraic equations, which determine the final analytical solutions. Comprehensive benchmark results for representative cylindrical shell panels with combinations of clamped and simply supported edges are presented, which are well validated by satisfactory agreement with other solution methods. Due to its rigorous and straightforward solution procedure, the developed method provides a solid easy-to-implement approach for exploring new analytical solutions.
\end{abstract}

\section{Introduction}

Shells are important load-bearing components with broad applications to engineering structures such as buildings, aircrafts, pressure vessels and launch vehicles. Accordingly, the mechanical behaviors of shells have attracted extensive investigations for many years (Leissa, 1973; Timoshenko and Woinowsky-Krieger, 1959). Whichever theory is adopted, the mechanics problems of shells are reduced to solving the governing high-order partial differential equations (PDEs) subject to specific boundary conditions. Therefore, a critical issue is to develop feasible solution approaches, which generally fall into two categories, $i$. e., analytical and numerical. Compared with numerical solution methods, analytical ones were less developed due to the acknowledged mathematical difficulty in treating the complex boundary-value problems of the PDEs in a rigorous way. While various effective numerical methods have been proposed for shell problems, it is still necessary to explore analytical methods, which can not only provide benchmark theoretical solutions of permanent significance but can also explicitly capture the relationships between mechanical responses and inputs, thus are especially useful for rapid structural analysis, efficient parameter optimization and design.
Among lots of numerical methods available in the literature, the representative ones include the finite element method (FEM) (Achryya et al., 2009), finite strip method (Assaee and Hasani, 2015), boundary element method (Dirgantara and Aliabadi, 2012), differential cubature method (Mousavi and Aghdam, 2009), meshless method (Ferreira et al., 2011), strip layer method (Chen et al., 2013), etc. Compared with the numerical methods, there are much fewer analytical/semi-analytical methods reported for solving shell problems. Cinefra et al. (2010) derived the closed-form solutions of free vibration problems of simply supported multilayered shells made of functionally graded material. The proposed variable kinematic model was able to obtain exact values and to establish the accuracy of classical shell theories, especially for multilayered shells. H. Li et al. (2019) investigated the free vibration of uniform and stepped annular-spherical shells with general boundary conditions by using the Rayleigh-Ritz approach, where the displacement functions of shell segments consisted of the Jacobi polynomials along the axial direction and the standard Fourier series along the circumferential direction. The results showed that the method has good convergence and excellent accuracy. R. Li et al. (2019) proposed an analytical symplectic superposition method for analyzing the mechanical behaviors of plate and shell structures (Zheng et al., 2019). The

\footnotetext{
* Corresponding author.

E-mail address: ruili@dlut.edu.cn (R. Li).
} 


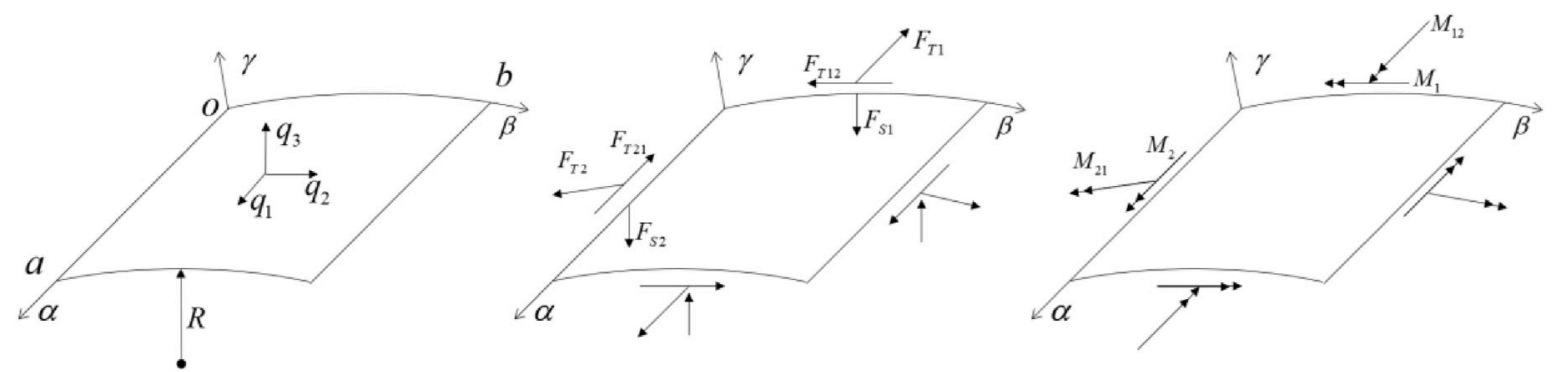

Fig. 1. A cylindrical shell panel in the $\alpha o \beta$ curvilinear coordinate system, with the directions of the positive external loads and internal forces indicated.

$\left(\frac{\partial^{2}}{\partial \alpha^{2}}+\frac{1-\mu}{2} \frac{\partial^{2}}{\partial \beta^{2}}\right) u+\frac{1+\mu}{2} \frac{\partial^{2} v}{\partial \alpha \partial \beta}+\frac{\mu}{R} \frac{\partial w}{\partial \alpha}=-\frac{1-\mu^{2}}{E \delta} q_{1}$

$\frac{1+\mu}{2} \frac{\partial^{2} u}{\partial \alpha \partial \beta}+\left(\frac{\partial^{2}}{\partial \beta^{2}}+\frac{1-\mu}{2} \frac{\partial^{2}}{\partial \alpha^{2}}\right) v+\frac{1}{R} \frac{\partial w}{\partial \beta}=-\frac{1-\mu^{2}}{E \delta} q_{2}$

$\frac{\mu}{R} \frac{\partial u}{\partial \alpha}+\frac{1}{R} \frac{\partial v}{\partial \beta}+\frac{w}{R^{2}}+\frac{\delta^{2}}{12} \nabla^{2} \nabla^{2} w=\frac{1-\mu^{2}}{E \delta} q_{3}$

method superposed several elaborated subproblems and was implemented within the Hamiltonian-system framework (Lim and Xu, 2010; Lim et al., 2009), showing rigorousness and high accuracy.

Integral transforms are a class of powerful methods that have found broad applications in solving the PDEs in various research fields such as heat transfer (Yang, 2016), convective diffusion (Cotta et al., 2013), nuclear physics (Ortakaya, 2012), fluid flow (Silva et al., 2011), signal process (Zhang et al., 2012), Tribology (Santos et al., 2012), and elasticity (Sneddon, 1975). Specifically, some recent progresses have been made on solving beam and plate problems by the finite integral transform method. An and Su (2011) employed the generalized finite integral transform technique to obtain a hybrid analytical-numerical solution for dynamic response of clamped axially moving beams. The approach can be either employed for benchmarking purposes or as an engineering simulation tool with exceptional computational performance. Matt (2013) combined classical and generalized finite integral transform methods for the transverse vibration analysis of a damaged simply supported beam. Numerical results obtained for a damaged beam demonstrated the accuracy, convergence and robustness of the combined strategy. Li et al. (2009) developed the finite integral transform method for exact bending solutions of fully clamped orthotropic rectangular thin plates, and extended it to buckling problems of thick plates (Ullah et al., 2019). Zhang et al. (2019) obtained new exact solutions for transverse vibration of rotationally-restrained orthotropic plates. Very recently, He et al. (2020a, 2020b) analyzed the free vibration of orthotropic rectangular thin plates with free edges and bending of the same plates with two opposite edges clamped by the generalized integral transform technique. Zhang et al. (2020) yielded the analytical free vibration solutions of orthotropic rectangular thin plates utilizing the similar technique. In spite of the advances in beam and plate analyses, there have been no reports, to the best of our knowledge, on extending such effective methods for analytical solutions of shell problems, which may be attributed to greater complexity of the shells.

In this study, we make the first attempt to further develop the finite integral transform method for analytical solutions of static problems of non-Lévy-type cylindrical shell panels without a free edge, i.e., those without two parallel edges simply supported, which cannot be obtained by a classical semi-inverse method. The current method is rigorous, straightforward, and with broad applicability, thus has great potential for accurate analysis of similar complex boundary-value problems of high-order PDEs. The rest of this paper is organized as follows. Section 2 presents the solution procedure based on three double finite integral transforms, which are imposed on the governing PDEs for bending of cylindrical shell panels. Inputting some boundary conditions, the relationship between the transforms of the displacements and specific unknowns is established. The inversions with those unknowns are substituted into the remaining boundary conditions to form infinite systems of linear simultaneous equations, the solutions of which finally yield the analytical solutions. Detailed derivations of fully clamped cylindrical shell panels serve as an example. Non-Lévy-type cylindrical shell panels with other combinations of clamped and simply supported edges are then solved in Section 3 to further demonstrate the generality of the developed method. In Section 4, comprehensive numerical results of cylindrical shell panels with different boundary conditions are tabulated in 11 tables, with 800 data listed for benchmark use, all of which are well validated by two available numerical and analytical methods. A convergence study is also implemented to consolidate the validity of the present solutions. Conclusions of the study are drawn in Section 5 as a closure.

\section{Solution procedure based on finite integral transforms}

Since this study focuses on cylindrical shell panels with combinations of clamped and simply supported edges, a convenient description of the boundary constraints is helpful. Using " $\mathrm{C}$ " and " $\mathrm{S}$ " as the abbreviations of "clamped" and "simply supported", a four-letter label is adopted to describe a panel according to the boundary constraints at the four edges, starting from the edge at $\beta=0$ (Fig. 1) and proceeding counterclockwise.

\subsection{Governing PDEs for bending of cylindrical shell panels}

According to the well-accepted Donnell-Mushtari theory (Leissa, 1973), the governing PDEs for bending of a cylindrical shell panel as shown in Fig. 1 can be presented in the $\alpha o \beta$ curvilinear coordinate system as.where $u, v$, and $w$ are the displacements along the $\alpha$ (longitudinal), $\beta$ (circumferential), and $\gamma$ (radial) directions, respectively, with $q_{1}$, $q_{2}$, and $q_{3}$ being the external load components in the corresponding directions; $R$ is the radius of curvature along the $\beta$ direction; $E$ is the Young's modulus; $\mu$ is the Poisson's ratio; $\nabla^{2}=\partial^{2} / \partial \alpha^{2}+\partial^{2} / \partial \beta^{2}$ is the Laplacian operator. The dimensions of the panel in the $\alpha, \beta$, and $\gamma$ directions are denoted by $a, b$, and $\delta$, respectively.

The displacements and internal forces are related by the following expressions: 
Table 1

Convergence for the displacement solutions of CCCC cylindrical shell panels.

\begin{tabular}{|c|c|c|c|c|c|c|c|c|}
\hline \multirow[t]{2}{*}{ Displacement } & \multirow[t]{2}{*}{ Location } & \multicolumn{5}{|c|}{ Number of series terms } & \multirow[t]{2}{*}{ SSM } & \multirow[t]{2}{*}{ FEM } \\
\hline & & 100 & 200 & 300 & 400 & 500 & & \\
\hline \multirow[t]{4}{*}{$\widehat{u}$} & $(a / 8, b / 4)$ & 0.01879 & 0.01888 & 0.01889 & 0.01887 & 0.01887 & 0.01887 & 0.01916 \\
\hline & $(a / 4, b / 8)$ & 0.01242 & 0.01244 & 0.01244 & 0.01244 & 0.01244 & 0.01242 & 0.01279 \\
\hline & $(a / 4,3 b / 8)$ & 0.03675 & 0.03678 & 0.03678 & 0.03678 & 0.03678 & 0.03678 & 0.03701 \\
\hline & $(3 a / 8, b / 4)$ & -0.007547 & -0.007557 & -0.007553 & -0.007554 & -0.007554 & -0.007554 & -0.007446 \\
\hline \multirow[t]{4}{*}{$\widehat{v}$} & $(a / 8, b / 4)$ & -0.02997 & -0.02996 & -0.02996 & -0.02996 & -0.02996 & -0.02998 & -0.02991 \\
\hline & $(a / 4, b / 8)$ & 0.3516 & 0.3528 & 0.3530 & 0.3529 & 0.3529 & 0.3528 & 0.3547 \\
\hline & $(a / 4,3 b / 8)$ & -0.05793 & -0.05830 & -0.05834 & -0.05832 & -0.05832 & -0.05831 & -0.05840 \\
\hline & $(3 a / 8, b / 4)$ & -0.07325 & -0.07319 & -0.07318 & -0.07318 & -0.07318 & -0.07327 & -0.07322 \\
\hline \multirow[t]{4}{*}{$\widehat{w}$} & $(a / 8, b / 4)$ & 9.626 & 9.628 & 9.629 & 9.629 & 9.629 & 9.629 & 9.638 \\
\hline & $(a / 4, b / 8)$ & 12.54 & 12.55 & 12.55 & 12.55 & 12.55 & 12.55 & 12.56 \\
\hline & $(a / 4,3 b / 8)$ & 8.486 & 8.488 & 8.487 & 8.487 & 8.487 & 8.487 & 8.488 \\
\hline & $(3 a / 8, b / 4)$ & 11.44 & 11.45 & 11.45 & 11.45 & 11.45 & 11.45 & 11.46 \\
\hline
\end{tabular}

Table 2

Convergence for the bending moment solutions of CCCC cylindrical shell panels.

\begin{tabular}{|c|c|c|c|c|c|c|c|c|}
\hline \multirow[t]{2}{*}{ Displacement } & \multirow[t]{2}{*}{ Location } & \multicolumn{5}{|c|}{ Number of series terms } & \multirow[t]{2}{*}{ SSM } & \multirow[t]{2}{*}{ FEM } \\
\hline & & 500 & 1000 & 1500 & 2000 & 2500 & & \\
\hline \multirow[t]{4}{*}{$\bar{M}_{1}$} & $(a / 8, b / 4)$ & -0.358 & -0.356 & -0.356 & -0.355 & -0.355 & -0.354 & -0.355 \\
\hline & $(a / 4, b / 8)$ & 0.424 & 0.454 & 0.461 & 0.471 & 0.471 & 0.480 & 0.480 \\
\hline & $(a / 4,3 b / 8)$ & -0.0981 & -0.0710 & -0.0650 & -0.0559 & -0.0559 & -0.0485 & -0.0485 \\
\hline & $(3 a / 8, b / 4)$ & 0.0359 & 0.0373 & 0.0376 & 0.0380 & 0.0380 & 0.0382 & 0.0376 \\
\hline \multirow[t]{4}{*}{$\bar{M}_{2}$} & $(a / 8, b / 4)$ & -0.348 & -0.340 & -0.339 & -0.336 & -0.336 & -0.334 & -0.344 \\
\hline & $(a / 4, b / 8)$ & 1.54 & 1.55 & 1.56 & 1.56 & 1.56 & 1.56 & 1.55 \\
\hline & $(a / 4,3 b / 8)$ & -0.157 & -0.148 & -0.147 & -0.144 & -0.144 & -0.142 & -0.150 \\
\hline & $(3 a / 8, b / 4)$ & 0.0134 & 0.0178 & 0.0187 & 0.0202 & 0.0202 & 0.0211 & 0.0100 \\
\hline
\end{tabular}

Table 3

Displacement solutions of CCCC cylindrical shell panels.

\begin{tabular}{|c|c|c|c|c|c|c|c|c|c|c|c|c|c|}
\hline \multirow[b]{2}{*}{$\alpha$} & \multirow[b]{2}{*}{$\beta$} & \multicolumn{4}{|l|}{$\widehat{u}$} & \multicolumn{4}{|l|}{$\widehat{v}$} & \multicolumn{4}{|l|}{$\widehat{w}$} \\
\hline & & $b / 8$ & $b / 4$ & $3 b / 8$ & $b / 2$ & $b / 8$ & $b / 4$ & $3 b / 8$ & $b / 2$ & $b / 8$ & $b / 4$ & $3 b / 8$ & $b / 2$ \\
\hline \multirow[t]{3}{*}{$a / 8$} & FEM & 0.01827 & 0.01916 & 0.05407 & 0.05937 & 0.2150 & -0.02991 & -0.02154 & 0 & 12.56 & 9.638 & 8.954 & 9.022 \\
\hline & SSM & 0.01779 & 0.01887 & 0.05374 & 0.05906 & 0.2133 & -0.02998 & -0.02145 & 0 & 12.54 & 9.629 & 8.953 & 9.019 \\
\hline & Present & 0.01782 & 0.01887 & 0.05374 & 0.05906 & 0.2134 & -0.02996 & -0.02145 & 0 & 12.54 & 9.629 & 8.953 & 9.019 \\
\hline \multirow[t]{3}{*}{$a / 4$} & FEM & 0.01279 & -0.005491 & 0.03701 & 0.04541 & 0.3547 & -0.06310 & -0.05840 & 0 & 12.56 & 10.57 & 8.488 & 8.835 \\
\hline & SSM & 0.01242 & -0.005698 & 0.03678 & 0.04521 & 0.3528 & -0.06314 & -0.05831 & 0 & 12.55 & 10.56 & 8.487 & 8.831 \\
\hline & Present & 0.01244 & -0.005698 & 0.03678 & 0.04521 & 0.3529 & -0.06308 & -0.05832 & 0 & 12.55 & 10.56 & 8.487 & 8.831 \\
\hline \multirow[t]{3}{*}{$3 a / 8$} & FEM & 0.006173 & -0.007446 & 0.01809 & 0.02505 & 0.4218 & -0.07322 & -0.09432 & 0 & 12.20 & 11.46 & 8.140 & 8.670 \\
\hline & SSM & 0.005984 & -0.007554 & 0.01797 & 0.02495 & 0.4199 & -0.07327 & -0.09422 & 0 & 12.18 & 11.45 & 8.140 & 8.665 \\
\hline & Present & 0.005995 & -0.007554 & 0.01797 & 0.02495 & 0.4200 & -0.07318 & -0.09424 & 0 & 12.18 & 11.45 & 8.140 & 8.665 \\
\hline \multirow[t]{3}{*}{$a / 2$} & FEM & 0 & 0 & 0 & 0 & 0.4417 & -0.07427 & -0.1090 & 0 & 12.05 & 11.78 & 8.025 & 8.590 \\
\hline & SSM & 0 & 0 & 0 & 0 & 0.4399 & -0.07432 & -0.1089 & 0 & 12.04 & 11.77 & 8.026 & 8.586 \\
\hline & Present & 0 & 0 & 0 & 0 & 0.4400 & -0.07422 & -0.1089 & 0 & 12.04 & 11.77 & 8.026 & 8.585 \\
\hline
\end{tabular}

$$
\begin{aligned}
& F_{T 1}=\frac{E \delta}{1-\mu^{2}}\left[\frac{\partial u}{\partial \alpha}+\mu\left(\frac{\partial v}{\partial \beta}+\frac{w}{R}\right)\right] \\
& F_{T 2}=\frac{E \delta}{1-\mu^{2}}\left(\frac{\partial v}{\partial \beta}+\frac{w}{R}+\mu \frac{\partial u}{\partial \alpha}\right) \\
& F_{T 12}=\frac{E \delta}{2(1+\mu)}\left(\frac{\partial u}{\partial \beta}+\frac{\partial v}{\partial \alpha}\right) \\
& M_{1}=-\frac{E \delta^{3}}{12\left(1-\mu^{2}\right)}\left(\frac{\partial^{2} w}{\partial \alpha^{2}}+\mu \frac{\partial^{2} w}{\partial \beta^{2}}\right) \\
& M_{2}=-\frac{E \delta^{3}}{12\left(1-\mu^{2}\right)}\left(\frac{\partial^{2} w}{\partial \beta^{2}}+\mu \frac{\partial^{2} w}{\partial \alpha^{2}}\right)
\end{aligned}
$$

$$
\begin{aligned}
& M_{12}=-\frac{E \delta^{3}}{12(1+\mu)}\left(\frac{\partial^{2} w}{\partial \alpha \partial \beta}\right) \\
& F_{S 1}=-\frac{E \delta^{3}}{12\left(1-\mu^{2}\right)} \frac{\partial}{\partial \alpha} \nabla^{2} w \\
& F_{S 2}=-\frac{E \delta^{3}}{12\left(1-\mu^{2}\right)} \frac{\partial}{\partial \beta} \nabla^{2} w
\end{aligned}
$$

where $F_{T 1}$ and $F_{T 2}$ are the in-plane forces that are normal to the $o \beta$ and $o \alpha$ edges; $F_{T 12}$ is the in-plane shear force; $F_{S 1}$ and $F_{S 2}$ are the transverse shear forces in the cross sections that are perpendicular to the $o \alpha$ and $o \beta$ edges; $M_{1}$ and $M_{2}$ are the bending moments about the $o \beta$ and $o \alpha$ edges; $M_{12}$ is the twisting moment. 
Table 4

Displacement solutions of CCCS cylindrical shell panels.

\begin{tabular}{|c|c|c|c|c|c|c|c|c|c|c|c|c|c|}
\hline \multirow[b]{2}{*}{$\alpha$} & \multirow[b]{2}{*}{$\beta$} & \multicolumn{4}{|l|}{$\widehat{u}$} & \multicolumn{4}{|l|}{$\widehat{v}$} & \multicolumn{4}{|l|}{$\widehat{w}$} \\
\hline & & $b / 8$ & $b / 4$ & $3 b / 8$ & $b / 2$ & $b / 8$ & $b / 4$ & $3 b / 8$ & $b / 2$ & $b / 8$ & $b / 4$ & $3 b / 8$ & $b / 2$ \\
\hline \multirow[t]{3}{*}{$a / 8$} & FEM & 0.5367 & 1.055 & 1.427 & 1.522 & 0.004785 & -0.3555 & -0.1474 & 0 & 15.43 & 9.317 & 8.141 & 9.119 \\
\hline & SSM & 0.5407 & 1.057 & 1.429 & 1.524 & 0.003268 & -0.3552 & -0.1475 & 0 & 15.43 & 9.287 & 8.133 & 9.110 \\
\hline & Present & 0.5343 & 1.052 & 1.425 & 1.520 & 0.003474 & -0.3552 & -0.1476 & 0 & 15.43 & 9.288 & 8.133 & 9.109 \\
\hline \multirow[t]{3}{*}{$a / 4$} & FEM & 0.4032 & 0.7872 & 1.101 & 1.188 & 0.01070 & -0.6137 & -0.2809 & 0 & 16.99 & 9.775 & 6.513 & 8.374 \\
\hline & SSM & 0.3986 & 0.7827 & 1.097 & 1.184 & 0.008871 & -0.6133 & -0.2811 & 0 & 17.00 & 9.746 & 6.505 & 8.363 \\
\hline & Present & 0.3986 & 0.7826 & 1.097 & 1.184 & 0.009131 & -0.6132 & -0.2812 & 0 & 16.99 & 9.749 & 6.505 & 8.362 \\
\hline \multirow[t]{3}{*}{$3 a / 8$} & FEM & 0.3118 & 0.5837 & 0.8074 & 0.8788 & 0.02697 & -0.7422 & -0.3749 & 0 & 17.44 & 10.48 & 5.464 & 7.769 \\
\hline & SSM & 0.3086 & 0.5799 & 0.8038 & 0.8753 & 0.02538 & -0.7416 & -0.3752 & 0 & 17.29 & 10.85 & 5.197 & 7.459 \\
\hline & Present & 0.3086 & 0.5798 & 0.8037 & 0.8752 & 0.02565 & -0.7415 & -0.3753 & 0 & 17.44 & 10.46 & 5.456 & 7.755 \\
\hline \multirow[t]{3}{*}{$a / 2$} & FEM & 0.2440 & 0.4256 & 0.5499 & 0.5996 & 0.05502 & -0.7533 & -0.4038 & 0 & 17.31 & 10.84 & 5.188 & 7.465 \\
\hline & SSM & 0.2457 & 0.4243 & 0.5515 & 0.6011 & 0.05369 & -0.7525 & -0.4040 & 0 & 17.28 & 10.84 & 5.191 & 7.445 \\
\hline & Present & 0.2434 & 0.4243 & 0.5484 & 0.5981 & 0.05395 & -0.7523 & -0.4041 & 0 & 17.28 & 10.84 & 5.191 & 7.444 \\
\hline \multirow[t]{3}{*}{$5 a / 8$} & FEM & 0.1899 & 0.2991 & 0.3327 & 0.3613 & 0.08441 & -0.6641 & -0.3600 & 0 & 16.75 & 10.68 & 5.683 & 7.522 \\
\hline & SSM & 0.1909 & 0.3003 & 0.3341 & 0.3625 & 0.08324 & -0.6631 & -0.3599 & 0 & 16.73 & 10.67 & 5.680 & 7.510 \\
\hline & Present & 0.1895 & 0.2983 & 0.3317 & 0.3601 & 0.08347 & -0.6629 & -0.3600 & 0 & 16.73 & 10.67 & 5.680 & 7.508 \\
\hline \multirow[t]{3}{*}{$3 a / 4$} & FEM & 0.1368 & 0.1850 & 0.1559 & 0.1700 & 0.09871 & -0.4880 & -0.2583 & 0 & 15.88 & 10.01 & 6.801 & 7.885 \\
\hline & SSM & 0.1361 & 0.1839 & 0.1544 & 0.1684 & 0.09765 & -0.4868 & -0.2579 & 0 & 15.85 & 10.01 & 6.801 & 7.877 \\
\hline & Present & 0.1360 & 0.1839 & 0.1544 & 0.1683 & 0.09785 & -0.4867 & -0.2580 & 0 & 15.85 & 10.01 & 6.801 & 7.876 \\
\hline \multirow[t]{3}{*}{$7 a / 8$} & FEM & 0.07198 & 0.06260 & 0.02122 & 0.02653 & 0.07049 & -0.2426 & -0.1297 & 0 & 14.27 & 9.434 & 8.213 & 8.496 \\
\hline & SSM & 0.07182 & 0.06218 & 0.02062 & 0.02579 & 0.06966 & -0.2413 & -0.1290 & 0 & 14.24 & 9.427 & 8.214 & 8.493 \\
\hline & Present & 0.07178 & 0.06218 & 0.02061 & 0.02576 & 0.06980 & -0.2413 & -0.1291 & 0 & 14.24 & 9.428 & 8.214 & 8.493 \\
\hline
\end{tabular}

Table 5

Displacement solutions of SCCC cylindrical shell panels.

\begin{tabular}{|c|c|c|c|c|c|c|c|c|c|c|c|c|c|}
\hline \multirow[b]{2}{*}{$\beta$} & \multirow[b]{2}{*}{$\alpha$} & \multicolumn{4}{|c|}{$\widehat{u}$} & \multicolumn{4}{|l|}{$\widehat{v}$} & \multicolumn{4}{|l|}{$\widehat{w}$} \\
\hline & & $a / 8$ & $a / 4$ & $3 a / 8$ & $a / 2$ & $a / 8$ & $a / 4$ & $3 a / 8$ & $a / 2$ & $a / 8$ & $a / 4$ & $3 a / 8$ & $a / 2$ \\
\hline \multirow[t]{3}{*}{$b / 8$} & FEM & -4.505 & -5.109 & -3.145 & 0 & 3.948 & 6.936 & 9.122 & 9.930 & 160.0 & 318.5 & 433.2 & 474.1 \\
\hline & SSM & -4.489 & -5.098 & -3.139 & 0 & 3.872 & 6.818 & 8.978 & 9.776 & 158.2 & 315.6 & 429.7 & 470.5 \\
\hline & Present & -4.485 & -5.095 & -3.138 & 0 & 3.873 & 6.817 & 8.976 & 9.774 & 158.1 & 315.4 & 429.5 & 470.3 \\
\hline \multirow[t]{3}{*}{$b / 4$} & FEM & -0.6031 & -0.8748 & -0.6208 & 0 & -3.188 & -8.580 & -13.10 & -14.83 & -0.02856 & -26.78 & -45.23 & -51.23 \\
\hline & SSM & -0.6016 & -0.8718 & -0.6186 & 0 & -3.165 & -8.525 & -13.03 & -14.74 & -0.01682 & -26.64 & -45.09 & -51.10 \\
\hline & Present & -0.6003 & -0.8705 & -0.6179 & 0 & -3.159 & -8.517 & -13.02 & -14.73 & 0.01230 & -26.62 & -45.09 & -51.10 \\
\hline \multirow[t]{3}{*}{$3 b / 8$} & FEM & 0.7632 & 0.9285 & 0.6080 & 0 & -0.1739 & -0.09141 & -0.01389 & 0.006368 & -12.06 & -42.68 & -70.26 & -81.3 \\
\hline & SSM & 0.7578 & 0.9223 & 0.6041 & 0 & -0.1747 & -0.09869 & -0.02543 & -0.006495 & -11.89 & -42.30 & -69.70 & -80.66 \\
\hline & Present & 0.7570 & 0.9218 & 0.6038 & 0 & -0.1745 & -0.09762 & -0.02356 & -0.004294 & -11.86 & -42.24 & -69.62 & -80.58 \\
\hline \multirow[t]{3}{*}{$b / 2$} & FEM & 0.03008 & 0.007910 & 0.000025 & 0 & 0.5848 & 1.510 & 2.312 & 2.628 & 13.54 & 23.21 & 32.23 & 35.84 \\
\hline & SSM & 0.03136 & 0.009612 & 0.001141 & 0 & 0.5794 & 1.498 & 2.295 & 2.608 & 13.48 & 23.02 & 31.93 & 35.50 \\
\hline & Present & 0.03122 & 0.009414 & 0.001006 & 0 & 0.5785 & 1.496 & 2.292 & 2.605 & 13.47 & 23.02 & 31.93 & 35.50 \\
\hline \multirow[t]{3}{*}{$5 b / 8$} & FEM & -0.07478 & -0.1223 & -0.08620 & 0 & -0.04839 & -0.2062 & -0.3561 & -0.4168 & 12.18 & 15.34 & 17.9 & 18.89 \\
\hline & SSM & -0.07419 & -0.1214 & -0.08562 & 0 & -0.04669 & -0.2006 & -0.3472 & -0.4065 & 12.15 & 15.31 & 17.86 & 18.85 \\
\hline & Present & -0.07399 & -0.1213 & -0.08553 & 0 & -0.04664 & -0.2006 & -0.3472 & -0.4066 & 12.14 & 15.30 & 17.85 & 18.83 \\
\hline \multirow[t]{3}{*}{$3 b / 4$} & FEM & 0.04358 & 0.02503 & 0.01274 & 0 & -0.06726 & -0.1634 & -0.2569 & -0.2949 & 8.281 & 6.788 & 5.534 & 5.015 \\
\hline & SSM & 0.04266 & 0.02407 & 0.01214 & 0 & -0.06633 & -0.1620 & -0.2552 & -0.2931 & 8.293 & 6.831 & 5.603 & 5.094 \\
\hline & Present & 0.04268 & 0.02409 & 0.01215 & 0 & -0.06626 & -0.1618 & -0.2550 & -0.2929 & 8.298 & 6.838 & 5.612 & 5.103 \\
\hline \multirow[t]{3}{*}{$7 b / 8$} & FEM & 0.03169 & 0.02906 & 0.01666 & 0 & -0.2070 & -0.3152 & -0.3524 & -0.3605 & 12.42 & 12.51 & 12.28 & 12.19 \\
\hline & SSM & 0.03115 & 0.02862 & 0.01643 & 0 & -0.2056 & -0.3145 & -0.3522 & -0.3605 & 12.40 & 12.48 & 12.24 & 12.15 \\
\hline & Present & 0.03123 & 0.02867 & 0.01645 & 0 & -0.2060 & -0.3149 & -0.3527 & -0.3610 & 12.40 & 12.48 & 12.24 & 12.15 \\
\hline
\end{tabular}

The non-Lévy-type cylindrical shell panels without a free edge include CCCC, CCCS, SCCC and SCCS cases, which will be treated successively in the following.

\subsection{Double finite integral transforms}

Three pairs of double finite integral transforms with respect to $u, v$, and $w$ are defined. The transforms are

$$
\begin{aligned}
& \bar{u}_{\mathrm{cs}}(m, n)=\int_{0}^{a} \int_{0}^{b} u(x, y) \cos \left(\lambda_{m} \alpha\right) \sin \left(\gamma_{n} \beta\right) \mathrm{d} \alpha \mathrm{d} \beta \\
& \bar{v}_{\mathrm{sc}}(m, n)=\int_{0}^{a} \int_{0}^{b} v(x, y) \sin \left(\lambda_{m} \alpha\right) \cos \left(\gamma_{n} \beta\right) \mathrm{d} \alpha \mathrm{d} \beta \\
& \bar{w}_{\mathrm{ss}}(m, n)=\int_{0}^{a} \int_{0}^{b} w(x, y) \sin \left(\lambda_{m} \alpha\right) \sin \left(\gamma_{n} \beta\right) \mathrm{d} \alpha d \beta
\end{aligned}
$$

$$
\begin{aligned}
& u(\alpha, \beta)=\frac{2}{a b} \sum_{m=0}^{\infty} \sum_{n=1}^{\infty} \varepsilon_{m} \bar{u}_{\mathrm{cs}}(m, n) \cos \left(\lambda_{m} \alpha\right) \sin \left(\gamma_{n} \beta\right) \\
& v(\alpha, \beta)=\frac{2}{a b} \sum_{m=1}^{\infty} \sum_{n=0}^{\infty} \varepsilon_{n} \bar{v}_{\mathrm{sc}}(m, n) \sin \left(\lambda_{m} \alpha\right) \cos \left(\gamma_{n} \beta\right) \\
& w(\alpha, \beta)=\frac{4}{a b} \sum_{m=1}^{\infty} \sum_{n=1}^{\infty} \bar{w}_{\mathrm{ss}}(m, n) \sin \left(\lambda_{m} \alpha\right) \sin \left(\gamma_{n} \beta\right)
\end{aligned}
$$

where $\lambda_{m}=m \pi / a, \gamma_{n}=n \pi / b, \varepsilon_{m}=\left\{\begin{array}{ll}1 & \text { for } m=0 \\ 2 & \text { for } m=1,2,3, \ldots\end{array}\right.$, and $\varepsilon_{n}=$ $\{1$ for $n=0$

2 for $n=1,2,3, \cdots$.

and the inversions are 
Table 6

Longitudinal displacement solutions, $\widehat{u}$, of SCCS cylindrical shell panels.

\begin{tabular}{|c|c|c|c|c|c|c|c|c|c|}
\hline$\alpha$ & $\beta$ & 0 & $b / 8$ & $b / 4$ & $3 b / 8$ & $b / 2$ & $5 b / 8$ & $3 b / 4$ & $7 b / 8$ \\
\hline \multirow[t]{3}{*}{0} & FEM & 0 & -14.22 & -4.685 & 2.204 & 1.33 & 0.5061 & 0.8215 & 0.6284 \\
\hline & SSM & 0 & -14.17 & -4.683 & 2.192 & 1.338 & 0.5117 & 0.8185 & 0.6243 \\
\hline & Present & 0 & -14.17 & -4.682 & 2.191 & 1.337 & 0.5115 & 0.8183 & 0.6243 \\
\hline \multirow[t]{3}{*}{$a / 8$} & FEM & 0 & -12.89 & -4.637 & 1.695 & 0.9637 & 0.2501 & 0.5308 & 0.4113 \\
\hline & SSM & 0 & -12.84 & -4.633 & 1.685 & 0.9709 & 0.2555 & 0.5284 & 0.4077 \\
\hline & Present & 0 & -12.84 & -4.632 & 1.684 & 0.9701 & 0.2553 & 0.5283 & 0.4078 \\
\hline \multirow[t]{3}{*}{$a / 4$} & FEM & 0 & -9.104 & -3.838 & 0.8433 & 0.5219 & 0.1098 & 0.3075 & 0.2621 \\
\hline & SSM & 0 & -9.067 & -3.832 & 0.8377 & 0.5275 & 0.1140 & 0.3061 & 0.2596 \\
\hline & Present & 0 & -9.065 & -3.832 & 0.8368 & 0.5269 & 0.1139 & 0.3059 & 0.2596 \\
\hline \multirow[t]{3}{*}{$3 a / 8$} & FEM & 0 & -4.185 & -2.394 & -0.1874 & 0.08433 & 0.08704 & 0.1733 & 0.1552 \\
\hline & SSM & 0 & -4.165 & -2.386 & -0.1867 & 0.08761 & 0.08939 & 0.1730 & 0.1539 \\
\hline & Present & 0 & -4.164 & -2.386 & -0.1875 & 0.08720 & 0.08928 & 0.1729 & 0.1539 \\
\hline \multirow[t]{3}{*}{$a / 2$} & FEM & 0 & 0.7792 & -0.7171 & -1.173 & -0.2884 & 0.1303 & 0.09980 & 0.07683 \\
\hline & SSM & 0 & 0.7822 & -0.7068 & -1.166 & -0.2877 & 0.1306 & 0.1006 & 0.07647 \\
\hline & Present & 0 & 0.7828 & -0.7072 & -1.167 & -0.2878 & 0.1305 & 0.1005 & 0.07645 \\
\hline \multirow[t]{3}{*}{$5 a / 8$} & FEM & 0 & 4.830 & 0.6653 & -1.849 & -0.5305 & 0.1780 & 0.06142 & 0.02073 \\
\hline & SSM & 0 & 4.819 & 0.6761 & -1.838 & -0.5318 & 0.1767 & 0.06297 & 0.02111 \\
\hline & Present & 0 & 4.818 & 0.6752 & -1.838 & -0.5317 & 0.1766 & 0.06291 & 0.02106 \\
\hline \multirow[t]{3}{*}{$3 a / 4$} & FEM & 0 & 6.920 & 1.307 & -1.973 & -0.5896 & 0.1775 & 0.03389 & -0.01481 \\
\hline & SSM & 0 & 6.900 & 1.316 & -1.960 & -0.5918 & 0.1754 & 0.03572 & -0.01395 \\
\hline & Present & 0 & 6.898 & 1.315 & -1.959 & -0.5915 & 0.1753 & 0.03566 & -0.01403 \\
\hline \multirow[t]{3}{*}{$7 a / 8$} & FEM & 0 & 5.730 & 1.018 & -1.383 & -0.4357 & 0.09827 & -0.007010 & -0.02864 \\
\hline & SSM & 0 & 5.711 & 1.024 & -1.373 & -0.4372 & 0.09669 & -0.005571 & -0.02773 \\
\hline & Present & 0 & 5.707 & 1.022 & -1.372 & -0.4368 & 0.09654 & -0.005620 & -0.02783 \\
\hline
\end{tabular}

Table 7

Circumferential displacement solutions, $\widehat{v}$, of SCCS cylindrical shell panels.

\begin{tabular}{|c|c|c|c|c|c|c|c|c|c|}
\hline$\alpha$ & $\beta$ & 0 & $b / 8$ & $b / 4$ & $3 b / 8$ & $b / 2$ & $5 b / 8$ & $3 b / 4$ & $7 b / 8$ \\
\hline \multirow[t]{3}{*}{0} & FEM & 0 & 0 & 0 & 0 & 0 & 0 & 0 & 0 \\
\hline & SSM & 0 & 0 & 0 & 0 & 0 & 0 & 0 & 0 \\
\hline & Present & 0 & 0 & 0 & 0 & 0 & 0 & 0 & 0 \\
\hline \multirow[t]{3}{*}{$a / 8$} & FEM & 35.25 & 4.444 & -11.34 & -1.787 & 2.172 & 0.1622 & -0.2303 & -0.08377 \\
\hline & SSM & 35.14 & 4.442 & -11.29 & -1.806 & 2.156 & 0.1721 & -0.2261 & -0.08346 \\
\hline & Present & 35.13 & 4.441 & -11.29 & -1.805 & 2.155 & 0.1717 & -0.2260 & -0.08420 \\
\hline \multirow[t]{3}{*}{$a / 4$} & FEM & 59.82 & 8.951 & -20.31 & -3.294 & 3.883 & 0.2942 & -0.4430 & -0.1602 \\
\hline & SSM & 59.61 & 8.921 & -20.22 & -3.330 & 3.854 & 0.3118 & -0.4354 & -0.1604 \\
\hline & Present & 59.60 & 8.919 & -20.21 & -3.327 & 3.853 & 0.3112 & -0.4354 & -0.1613 \\
\hline \multirow[t]{3}{*}{$3 a / 8$} & FEM & 72.89 & 12.16 & -25.07 & -4.239 & 4.760 & 0.3693 & -0.5840 & -0.2310 \\
\hline & SSM & 72.63 & 12.10 & -24.95 & -4.283 & 4.725 & 0.3910 & -0.5739 & -0.2317 \\
\hline & Present & 72.62 & 12.09 & -24.95 & -4.280 & 4.723 & 0.3903 & -0.5739 & -0.2326 \\
\hline \multirow[t]{3}{*}{$a / 2$} & FEM & 74.52 & 13.21 & -24.92 & -4.400 & 4.620 & 0.3759 & -0.5984 & -0.2912 \\
\hline & SSM & 74.24 & 13.13 & -24.81 & -4.441 & 4.584 & 0.3973 & -0.5884 & -0.2919 \\
\hline & Present & 74.22 & 13.12 & -24.80 & -4.439 & 4.582 & 0.3965 & -0.5885 & -0.2927 \\
\hline \multirow[t]{3}{*}{$5 a / 8$} & FEM & 65.49 & 11.96 & -20.30 & -3.7900 & 3.571 & 0.3192 & -0.4831 & -0.3250 \\
\hline & SSM & 65.23 & 11.89 & -20.20 & -3.819 & 3.542 & 0.3363 & -0.4744 & -0.3250 \\
\hline & Present & 65.22 & 11.89 & -20.19 & -3.817 & 3.540 & 0.3357 & -0.4745 & -0.3257 \\
\hline \multirow[t]{3}{*}{$3 a / 4$} & FEM & 47.54 & 8.905 & -12.67 & -2.674 & 2.029 & 0.2164 & -0.2877 & -0.3096 \\
\hline & SSM & 47.33 & 8.846 & -12.60 & -2.686 & 2.011 & 0.2268 & -0.2816 & -0.3086 \\
\hline & Present & 47.31 & 8.845 & -12.59 & -2.684 & 2.009 & 0.2263 & -0.2816 & -0.3092 \\
\hline \multirow[t]{3}{*}{$7 a / 8$} & FEM & 23.72 & 4.889 & -4.657 & -1.361 & 0.6045 & 0.09829 & -0.1026 & -0.2073 \\
\hline & SSM & 23.56 & 4.839 & -4.625 & -1.357 & 0.5987 & 0.1019 & -0.09953 & -0.2056 \\
\hline & Present & 23.55 & 4.840 & -4.619 & -1.357 & 0.5974 & 0.1016 & -0.09953 & -0.2060 \\
\hline
\end{tabular}

\subsection{Solution procedure with applications to fully clamped cylindrical} shell panels

Applying the three transforms in Eq. (12) over Eqs. (1)-(3), respectively, we obtain

$$
\begin{aligned}
& \int_{0}^{b} {\left.\left[\frac{\partial u}{\partial \alpha} \cos \left(\lambda_{m} \alpha\right)\right]\right|_{0} ^{a} \sin \left(\gamma_{n} \beta\right) \mathrm{d} \beta-\left.\frac{1-\mu}{2} \gamma_{n} \int_{0}^{b}\left[u \cos \left(\lambda_{m} \alpha\right)\right]\right|_{0} ^{a} \cos \left(\gamma_{n} \beta\right) \mathrm{d} \beta } \\
&-\left(\lambda_{m}^{2}+\frac{1-\mu}{2} \gamma_{n}^{2}\right) \bar{u}_{\mathrm{cs}}-\frac{1+\mu}{2}\left\{\left.\gamma_{n} \int_{0}^{b}\left[v \cos \left(\lambda_{m} \alpha\right)\right]\right|_{0} ^{a} \cos \left(\gamma_{n} \beta\right) \mathrm{d} \beta+\lambda_{m} \gamma_{n} \overline{\mathrm{sc}}_{\mathrm{sc}}\right\} \\
&+\frac{\mu}{R}\left\{\left.\int_{0}^{b}\left[w \cos \left(\lambda_{m} \alpha\right)\right]\right|_{0} ^{a} \sin \left(\gamma_{n} \beta\right) \mathrm{d} \beta+\lambda_{m} \bar{w}_{\mathrm{ss}}\right\}=-\frac{1-\mu^{2}}{E \delta} \bar{q}_{1 \mathrm{cs}}
\end{aligned}
$$

$\left.\int_{0}^{a}\left[\frac{\partial \nu}{\partial \beta} \cos \left(\gamma_{n} \beta\right)\right]\right|_{0} ^{b} \sin \left(\lambda_{m} \alpha\right) \mathrm{d} \alpha-\left.\frac{1-\mu}{2} \lambda_{m} \int_{0}^{a}\left[v \cos \left(\gamma_{n} \beta\right)\right]\right|_{0} ^{b} \cos \left(\lambda_{m} \alpha\right) \mathrm{d} \alpha$ $-\frac{1+\mu}{2}\left\{\left.\lambda_{m} \int_{0}^{a}\left[u \cos \left(\gamma_{n} \beta\right)\right]\right|_{0} ^{b} \cos \left(\lambda_{m} \alpha\right) \mathrm{d} \alpha+\lambda_{m} \gamma_{n} \bar{u}_{\mathrm{cs}}\right\}-\left(\frac{1-\mu}{2} \lambda_{m}^{2}+\gamma_{n}^{2}\right) \bar{v}_{\mathrm{sc}}$ $+\frac{1}{R}\left\{\left.\int_{0}^{a}\left[w \cos \left(\gamma_{n} \beta\right)\right]\right|_{0} ^{b} \sin \left(\lambda_{m} \alpha\right) \mathrm{d} \alpha+\gamma_{n} \bar{w}_{\mathrm{ss}}\right\}=-\frac{1-\mu^{2}}{E \delta} \bar{q}_{2 \mathrm{sc}}$ 
Table 8

Radial displacement solutions, $\widehat{w}$, of SCCS cylindrical shell panels.

\begin{tabular}{|c|c|c|c|c|c|c|c|c|c|}
\hline$\alpha$ & $\beta$ & 0 & $b / 8$ & $b / 4$ & $3 b / 8$ & $b / 2$ & $5 b / 8$ & $3 b / 4$ & $7 b / 8$ \\
\hline \multirow[t]{3}{*}{0} & FEM & 0 & 0 & 0 & 0 & 0 & 0 & 0 & 0 \\
\hline & SSM & 0 & 0 & 0 & 0 & 0 & 0 & 0 & 0 \\
\hline & Present & 0 & 0 & 0 & 0 & 0 & 0 & 0 & 0 \\
\hline \multirow[t]{3}{*}{$a / 8$} & FEM & 0 & 294.2 & -19.16 & -64.26 & 16.39 & 23.02 & 5.952 & 13.04 \\
\hline & SSM & 0 & 293.0 & -18.71 & -63.91 & 16.13 & 22.95 & 6.036 & 13.01 \\
\hline & Present & 0 & 293.0 & -18.72 & -63.89 & 16.13 & 22.94 & 6.039 & 13.02 \\
\hline \multirow[t]{3}{*}{$a / 4$} & FEM & 0 & 507.8 & -34.45 & -125.0 & 21.59 & 33.04 & 3.821 & 12.62 \\
\hline & SSM & 0 & 505.7 & -33.65 & -124.3 & 21.12 & 32.92 & 3.971 & 12.59 \\
\hline & Present & 0 & 505.6 & -33.67 & -124.3 & 21.13 & 32.91 & 3.977 & 12.59 \\
\hline \multirow[t]{3}{*}{$3 a / 8$} & FEM & 0 & 620.9 & -36.14 & -159.1 & 24.03 & 38.00 & 3.274 & 11.81 \\
\hline & SSM & 0 & 618.2 & -35.18 & -158.3 & 23.46 & 37.86 & 3.459 & 11.78 \\
\hline & Present & 0 & 618.1 & -35.19 & -158.2 & 23.47 & 37.84 & 3.466 & 11.77 \\
\hline \multirow[t]{3}{*}{$a / 2$} & FEM & 0 & 629.9 & -31.26 & -158.7 & 22.76 & 37.16 & 3.984 & 11.31 \\
\hline & SSM & 0 & 627.1 & -30.34 & -157.9 & 22.19 & 37.02 & 4.167 & 11.28 \\
\hline & Present & 0 & 627.0 & -30.35 & -157.8 & 22.20 & 37.00 & 4.174 & 11.28 \\
\hline \multirow[t]{3}{*}{$5 a / 8$} & FEM & 0 & 543.8 & -22.82 & -126.1 & 18.05 & 31.26 & 5.522 & 11.33 \\
\hline & SSM & 0 & 541.3 & -22.11 & -125.4 & 17.61 & 31.13 & 5.667 & 11.32 \\
\hline & Present & 0 & 541.1 & -22.10 & -125.4 & 17.61 & 31.12 & 5.674 & 11.31 \\
\hline \multirow[t]{3}{*}{$3 a / 4$} & FEM & 0 & 382.5 & -8.702 & -74.49 & 11.77 & 22.54 & 7.230 & 11.85 \\
\hline & SSM & 0 & 380.6 & -8.322 & -74.00 & 11.52 & 22.44 & 7.316 & 11.83 \\
\hline & Present & 0 & 380.5 & -8.303 & -73.95 & 11.51 & 22.43 & 7.322 & 11.83 \\
\hline \multirow[t]{3}{*}{$7 a / 8$} & FEM & 0 & 185.6 & 9.953 & -23.82 & 7.156 & 14.14 & 8.664 & 12.17 \\
\hline & SSM & 0 & 184.4 & 9.981 & -23.57 & 7.090 & 14.10 & 8.691 & 12.16 \\
\hline & Present & 0 & 184.3 & 10.01 & -23.54 & 7.087 & 14.09 & 8.695 & 12.16 \\
\hline
\end{tabular}

Table 9

Bending moment solutions of CCCC cylindrical shell panels.

\begin{tabular}{|c|c|c|c|c|c|c|c|c|c|c|c|}
\hline \multirow[b]{2}{*}{$\alpha$} & \multirow[b]{2}{*}{$\beta$} & \multicolumn{5}{|l|}{$\widehat{M}_{1}$} & \multicolumn{5}{|l|}{$\widehat{M}_{2}$} \\
\hline & & 0 & $b / 8$ & $b / 4$ & $3 b / 8$ & $b / 2$ & 0 & $b / 8$ & $b / 4$ & $3 b / 8$ & $b / 2$ \\
\hline \multirow[t]{3}{*}{0} & FEM & 0.719 & -30.9 & -27.9 & -27.4 & -27.3 & 0.715 & -9.32 & -8.42 & -8.26 & -8.23 \\
\hline & SSM & 0 & -31.1 & -28.1 & -27.6 & -27.5 & 0 & -9.32 & -8.43 & -8.28 & -8.25 \\
\hline & Present & 0 & -31.1 & -28.2 & -27.6 & -27.6 & 0 & -9.34 & -8.45 & -8.29 & -8.27 \\
\hline \multirow[t]{3}{*}{$a / 8$} & FEM & -2.52 & 0.393 & -0.355 & -0.216 & -0.215 & -8.39 & 1.37 & -0.344 & -0.0988 & -0.0572 \\
\hline & SSM & -2.52 & 0.393 & -0.354 & -0.216 & -0.216 & -8.38 & 1.38 & -0.334 & -0.0901 & -0.0512 \\
\hline & Present & -2.52 & 0.393 & -0.355 & -0.216 & -0.216 & -8.39 & 1.38 & -0.336 & -0.0901 & -0.0495 \\
\hline \multirow[t]{3}{*}{$a / 4$} & FEM & -1.62 & 0.480 & -0.0554 & -0.0485 & 0.0259 & -5.40 & 1.55 & -0.219 & -0.150 & 0.0738 \\
\hline & SSM & -1.61 & 0.480 & -0.0548 & -0.0485 & 0.0248 & -5.38 & 1.56 & -0.209 & -0.142 & 0.0791 \\
\hline & Present & -1.62 & 0.471 & -0.0627 & -0.0559 & 0.0177 & -5.38 & 1.56 & -0.212 & -0.144 & 0.0778 \\
\hline \multirow[t]{3}{*}{$3 a / 8$} & FEM & -1.33 & 0.391 & 0.0376 & -0.100 & 0.0407 & -4.45 & 1.34 & 0.0100 & -0.296 & 0.143 \\
\hline & SSM & -1.33 & 0.391 & 0.0382 & -0.0993 & 0.0394 & -4.43 & 1.35 & 0.0211 & -0.287 & 0.147 \\
\hline & Present & -1.33 & 0.391 & 0.0380 & -0.0992 & 0.0398 & -4.43 & 1.35 & 0.0202 & -0.287 & 0.148 \\
\hline \multirow[t]{3}{*}{$a / 2$} & FEM & -1.26 & 0.365 & 0.0678 & -0.115 & 0.0436 & -4.20 & 1.26 & 0.102 & -0.353 & 0.168 \\
\hline & SSM & -1.25 & 0.365 & 0.0684 & -0.115 & 0.0422 & -4.18 & 1.27 & 0.113 & -0.343 & 0.172 \\
\hline & Present & -1.25 & 0.371 & 0.0737 & -0.110 & 0.0478 & -4.18 & 1.27 & 0.114 & -0.342 & 0.175 \\
\hline
\end{tabular}

$-\frac{\mu}{R} \lambda_{m} \bar{u}_{\mathrm{cs}}-\frac{\gamma_{n}}{R} \bar{v}_{\mathrm{sc}}+\left[\frac{1}{R^{2}}+\frac{\delta^{2}}{12}\left(\lambda_{m}^{2}+\gamma_{n}^{2}\right)^{2}\right] \bar{w}_{\mathrm{ss}}$

$+\frac{\delta^{2}}{12}\left\{\left.\lambda_{m}^{3} \int_{0}^{b}\left[w \cos \left(\lambda_{m} \alpha\right)\right]\right|_{0} ^{a} \sin \left(\gamma_{n} \beta\right) \mathrm{d} \beta+\left.\lambda_{m}^{2} \gamma_{n} \int_{0}^{a}\left[w \cos \left(\gamma_{n} \beta\right)\right]\right|_{0} ^{b} \sin \left(\lambda_{m} \alpha\right) \mathrm{d} \alpha\right.$

$\left.+\left.\lambda_{m} \gamma_{n}^{2} \int_{0}^{b}\left[w \cos \left(\lambda_{m} \alpha\right)\right]\right|_{0} ^{a} \sin \left(\gamma_{n} \beta\right) \mathrm{d} \beta+\left.\gamma_{n}^{3} \int_{0}^{a}\left[w \cos \left(\gamma_{n} \beta\right)\right]\right|_{0} ^{b} \sin \left(\lambda_{m} \alpha\right) \mathrm{d} \alpha\right\}$

$-\frac{\delta^{2}}{12}\left\{\left.\lambda_{m} \int_{0}^{b}\left[\frac{\partial^{2} w}{\partial \alpha^{2}} \cos \left(\lambda_{m} \alpha\right)\right]\right|_{0} ^{a} \sin \left(\gamma_{n} \beta\right) \mathrm{d} \beta+\left.\gamma_{n} \int_{0}^{a}\left[\frac{\partial^{2} w}{\partial \beta^{2}} \cos \left(\gamma_{n} \beta\right)\right]\right|_{0} ^{b} \sin \left(\lambda_{m} \alpha\right) \mathrm{d} \alpha\right\}$

$\frac{1-\mu^{2}}{E \delta} \bar{q}_{3 \mathrm{ss}}$

(16)

where $\bar{q}_{1 \mathrm{cs}}, \bar{q}_{2 \mathrm{sc}}$, and $\bar{q}_{3 \mathrm{ss}}$ are the transforms of the external load components as defined for those of $u, v$, and $w$ in Eq. (12).

Without loss of generality, we first focus on CCCC panels. The boundary conditions are

$$
\begin{aligned}
& \left.\left(u, v, w, \frac{\partial w}{\partial \alpha}\right)\right|_{\alpha=0}=0 \\
& \left.\left(u, v, w, \frac{\partial w}{\partial \alpha}\right)\right|_{\alpha=a}=0 \\
& \left.\left(u, v, w, \frac{\partial w}{\partial \beta}\right)\right|_{\beta=0}=0 \\
& \left.\left(u, v, w, \frac{\partial w}{\partial \beta}\right)\right|_{\beta=b}=0
\end{aligned}
$$

Substituting the boundary conditions $\left.v\right|_{\alpha=0, a}=0,\left.u\right|_{\beta=0, b}=0$, $\left.w\right|_{\alpha=0, a}=0$, and $\left.w\right|_{\beta=0, b}=0$ into Eqs. (14)-(16) yields

$\left.\int_{0}^{b}\left[\frac{\partial u}{\partial \alpha} \cos \left(\lambda_{m} \alpha\right)\right]\right|_{0} ^{a} \sin \left(\gamma_{n} \beta\right) \mathrm{d} \beta-\left(\lambda_{m}^{2}+\frac{1-\mu}{2} \gamma_{n}^{2}\right) \bar{u}_{\mathrm{cs}}$

$-\frac{1+\mu}{2} \lambda_{m} \gamma_{n} \bar{v}_{\mathrm{sc}}+\frac{\mu}{R} \lambda_{m} \bar{w}_{\mathrm{ss}}=-\frac{1-\mu^{2}}{E \delta} \bar{q}_{1 \mathrm{cs}}$ 
Table 10

Bending moment solutions of CCCS cylindrical shell panels.

\begin{tabular}{|c|c|c|c|c|c|c|c|c|c|c|c|}
\hline \multirow[b]{2}{*}{$\alpha$} & \multirow[b]{2}{*}{$\beta$} & \multicolumn{5}{|l|}{$\widehat{M}_{1}$} & \multicolumn{5}{|l|}{$\widehat{M}_{2}$} \\
\hline & & 0 & $b / 8$ & $b / 4$ & $3 b / 8$ & $b / 2$ & 0 & $b / 8$ & $b / 4$ & $3 b / 8$ & $b / 2$ \\
\hline \multirow[t]{3}{*}{0} & FEM & -1.14 & 0.148 & 0.178 & 0.183 & 0.184 & 1.87 & 0.00511 & 0.0294 & 0.0330 & 0.0340 \\
\hline & SSM & 0 & 0 & 0 & 0 & 0 & 0 & 0 & 0 & 0 & 0 \\
\hline & Present & 0 & 0 & 0 & 0 & 0 & 0 & 0 & 0 & 0 & 0 \\
\hline \multirow[t]{3}{*}{$a / 8$} & FEM & -3.25 & 0.611 & -0.408 & -0.218 & -0.130 & -10.8 & 1.98 & -0.602 & -0.173 & 0.106 \\
\hline & SSM & -3.26 & 0.613 & -0.410 & -0.218 & -0.132 & -10.9 & 1.99 & -0.595 & -0.165 & 0.111 \\
\hline & Present & -3.26 & 0.617 & -0.407 & -0.215 & -0.129 & -10.9 & 1.99 & -0.597 & -0.164 & 0.114 \\
\hline \multirow[t]{3}{*}{$a / 4$} & FEM & -2.66 & 0.791 & -0.193 & -0.130 & 0.0930 & -8.86 & 2.46 & -0.614 & -0.328 & 0.331 \\
\hline & SSM & -2.66 & 0.794 & -0.195 & -0.131 & 0.0910 & -8.87 & 2.47 & -0.608 & -0.320 & 0.334 \\
\hline & Present & -2.66 & 0.793 & -0.197 & -0.132 & 0.0907 & -8.88 & 2.47 & -0.611 & -0.321 & 0.335 \\
\hline \multirow[t]{3}{*}{$3 a / 8$} & FEM & -2.41 & 0.770 & -0.112 & -0.198 & 0.122 & -8.02 & 2.46 & -0.445 & -0.527 & 0.451 \\
\hline & SSM & -2.41 & 0.772 & -0.112 & -0.199 & 0.120 & -8.02 & 2.47 & -0.437 & -0.521 & 0.454 \\
\hline & Present & -2.41 & 0.769 & -0.115 & -0.202 & 0.118 & -8.02 & 2.47 & -0.439 & -0.522 & 0.454 \\
\hline \multirow[t]{3}{*}{$a / 2$} & FEM & -2.26 & 0.738 & -0.0694 & -0.215 & 0.121 & -7.54 & 2.38 & -0.339 & -0.590 & 0.465 \\
\hline & SSM & -2.26 & 0.739 & -0.0686 & -0.216 & 0.119 & -7.53 & 2.39 & -0.329 & -0.584 & 0.468 \\
\hline & Present & -2.26 & 0.742 & -0.0662 & -0.213 & 0.122 & -7.54 & 2.40 & -0.329 & -0.584 & 0.470 \\
\hline \multirow[t]{3}{*}{$5 a / 8$} & FEM & -2.22 & 0.711 & -0.0797 & -0.179 & 0.0985 & -7.40 & 2.31 & -0.369 & -0.488 & 0.373 \\
\hline & SSM & -2.22 & 0.711 & -0.0786 & -0.180 & 0.0964 & -7.38 & 2.32 & -0.358 & -0.482 & 0.375 \\
\hline & Present & -2.22 & 0.720 & -0.0800 & -0.180 & 0.100 & -7.39 & 2.32 & -0.358 & -0.481 & 0.377 \\
\hline \multirow[t]{3}{*}{$3 a / 4$} & FEM & -2.36 & 0.708 & -0.146 & -0.0919 & 0.0526 & -7.85 & 2.24 & -0.495 & -0.263 & 0.203 \\
\hline & SSM & -2.35 & 0.707 & -0.144 & -0.0925 & 0.0508 & -7.83 & 2.25 & -0.483 & -0.257 & 0.206 \\
\hline & Present & -2.35 & 0.700 & -0.152 & -0.0988 & 0.0449 & -7.84 & 2.25 & -0.487 & -0.259 & 0.206 \\
\hline \multirow[t]{3}{*}{$7 a / 8$} & FEM & -3.06 & 0.473 & -0.400 & -0.228 & -0.220 & -10.2 & 1.66 & -0.466 & -0.142 & -0.0199 \\
\hline & SSM & -3.06 & 0.472 & -0.399 & -0.228 & -0.221 & -10.2 & 1.67 & -0.455 & -0.134 & -0.0149 \\
\hline & Present & -3.06 & 0.470 & -0.402 & -0.230 & -0.222 & -10.2 & 1.67 & -0.458 & -0.135 & -0.0138 \\
\hline \multirow[t]{3}{*}{$a$} & FEM & 0.687 & -32.4 & -28.3 & -27.3 & -27.3 & 0.684 & -9.76 & -8.53 & -8.23 & -8.22 \\
\hline & SSM & 0 & -32.6 & -28.4 & -27.5 & -27.5 & 0 & -9.69 & -8.61 & -8.25 & -8.21 \\
\hline & Present & 0 & -32.6 & -28.5 & -27.6 & -27.6 & 0 & -9.77 & -8.56 & -8.27 & -8.27 \\
\hline
\end{tabular}

Table 11

Bending moment solutions of SCCC cylindrical shell panels.

\begin{tabular}{|c|c|c|c|c|c|c|c|c|c|c|c|}
\hline \multirow[b]{2}{*}{$\beta$} & \multirow[b]{2}{*}{$\alpha$} & \multicolumn{5}{|l|}{$\widehat{M}_{1}$} & \multicolumn{5}{|l|}{$\widehat{M}_{2}$} \\
\hline & & 0 & $a / 8$ & $a / 4$ & $3 a / 8$ & $a / 2$ & 0 & $a / 8$ & $a / 4$ & $3 a / 8$ & $a / 2$ \\
\hline \multirow[t]{3}{*}{0} & FEM & 16.5 & -0.209 & -0.160 & -0.145 & -0.141 & -10.1 & -0.0830 & 0 & 0.0335 & 0.0419 \\
\hline & SSM & 0 & 0 & 0 & 0 & 0 & 0 & 0 & 0 & 0 & 0 \\
\hline & Present & 0 & 0 & 0 & 0 & 0 & 0 & 0 & 0 & 0 & 0 \\
\hline \multirow[t]{3}{*}{$b / 8$} & FEM & -136 & 2.64 & 14.9 & 22.5 & 25.1 & -41.1 & 14.1 & 41.4 & 61.7 & 68.9 \\
\hline & SSM & -134 & 2.62 & 14.8 & 22.5 & 25.0 & -40.3 & 14.0 & 41.2 & 61.5 & 68.7 \\
\hline & Present & -135 & 2.61 & 14.7 & 22.4 & 25.1 & -40.6 & 14.0 & 41.1 & 61.5 & 68.7 \\
\hline \multirow[t]{3}{*}{$b / 4$} & FEM & -37.5 & -3.01 & -8.81 & -12.5 & -13.7 & -11.4 & -11.1 & -27.6 & -39.6 & -43.6 \\
\hline & SSM & -37.4 & -2.96 & -8.73 & -12.5 & -13.6 & -11.2 & -11.0 & -27.4 & -39.3 & -43.3 \\
\hline & Present & -37.8 & -2.96 & -8.76 & -12.5 & -13.6 & -11.3 & -11.0 & -27.4 & -39.3 & -43.3 \\
\hline \multirow[t]{3}{*}{$3 b / 8$} & FEM & -14.3 & 0.0292 & -1.01 & -2.36 & -2.96 & -4.31 & -1.40 & -2.83 & -4.79 & -5.78 \\
\hline & SSM & -14.7 & 0.0229 & -1.01 & -2.36 & -2.96 & -4.40 & -1.40 & -2.84 & -4.81 & -5.78 \\
\hline & Present & -14.8 & 0.0237 & -1.02 & -2.35 & -2.94 & -4.44 & -1.40 & -2.84 & -4.79 & -5.77 \\
\hline \multirow[t]{3}{*}{$b / 2$} & FEM & -27.8 & 0.109 & 1.70 & 2.96 & 3.47 & -8.39 & 1.92 & 5.53 & 8.86 & 10.2 \\
\hline & SSM & -28.0 & 0.105 & 1.69 & 2.94 & 3.44 & -8.41 & 1.91 & 5.49 & 8.79 & 10.1 \\
\hline & Present & -28.3 & 0.105 & 1.66 & 2.94 & 3.46 & -8.48 & 1.91 & 5.48 & 8.79 & 10.1 \\
\hline \multirow[t]{3}{*}{$5 b / 8$} & FEM & -29.8 & -0.271 & -0.120 & -0.241 & -0.293 & -9.00 & -0.114 & -0.524 & -1.10 & -1.35 \\
\hline & SSM & -30.0 & -0.269 & -0.114 & -0.232 & -0.282 & -9.00 & -0.100 & -0.498 & -1.06 & -1.30 \\
\hline & Present & -30.3 & -0.270 & -0.140 & -0.233 & -0.266 & -9.08 & -0.103 & -0.508 & -1.06 & -1.30 \\
\hline \multirow[t]{3}{*}{$3 b / 4$} & FEM & -27.5 & -0.418 & -0.372 & -0.483 & -0.529 & -8.28 & -0.745 & -1.22 & -1.48 & -1.58 \\
\hline & SSM & -27.7 & -0.417 & -0.369 & -0.479 & -0.525 & -8.30 & -0.731 & -1.20 & -1.46 & -1.56 \\
\hline & Present & -27.9 & -0.419 & -0.393 & -0.479 & -0.508 & -8.37 & -0.736 & -1.21 & -1.46 & -1.55 \\
\hline \multirow[t]{3}{*}{$7 b / 8$} & FEM & -30.6 & 0.429 & 0.609 & 0.604 & 0.611 & -9.24 & 1.52 & 1.99 & 2.04 & 2.06 \\
\hline & SSM & -30.8 & 0.428 & 0.606 & 0.600 & 0.607 & -9.24 & 1.52 & 1.99 & 2.03 & 2.05 \\
\hline & Present & -31.1 & 0.429 & 0.582 & 0.601 & 0.625 & -9.24 & 1.52 & 1.99 & 2.03 & 2.06 \\
\hline \multirow[t]{3}{*}{$b$} & FEM & 0.720 & -2.57 & -1.80 & -1.63 & -1.59 & 0.715 & -8.55 & -5.99 & -5.42 & -5.32 \\
\hline & SSM & 0 & -2.57 & -1.75 & -1.63 & -1.60 & 0 & -8.53 & -6.00 & -5.39 & -5.27 \\
\hline & Present & 0 & -2.57 & -1.80 & -1.62 & -1.59 & 0 & -8.58 & -5.98 & -5.41 & -5.30 \\
\hline
\end{tabular}

$\left.\int_{0}^{a}\left[\frac{\partial v}{\partial \beta} \cos \left(\gamma_{n} \beta\right)\right]\right|_{0} ^{b} \sin \left(\lambda_{m} \alpha\right) \mathrm{d} x-\frac{1+\mu}{2} \lambda_{m} \gamma_{n} \bar{u}_{\mathrm{cs}}$

$-\left(\frac{1-\mu}{2} \lambda_{m}^{2}+\gamma_{n}^{2}\right) \bar{v}_{\mathrm{sc}}+\frac{1}{R} \gamma_{n} \bar{w}_{\mathrm{ss}}=-\frac{1-\mu^{2}}{E \delta} \bar{q}_{2 \mathrm{sc}}$ 
Table 12

Bending moment solutions, $\widehat{M}_{1}$, of SCCS cylindrical shell panels.

\begin{tabular}{|c|c|c|c|c|c|c|c|c|c|c|}
\hline \multirow[b]{2}{*}{$\alpha$} & \multirow[b]{2}{*}{$\beta$} & \multicolumn{9}{|l|}{$\widehat{M}_{1}$} \\
\hline & & 0 & $b / 8$ & $b / 4$ & $3 b / 8$ & $b / 2$ & $5 b / 8$ & $3 b / 4$ & $7 b / 8$ & $b$ \\
\hline \multirow[t]{3}{*}{0} & FEM & 0.0264 & 0.187 & 0.187 & 0.187 & 0.187 & 0.187 & 0.187 & 0.187 & 0.079 \\
\hline & SSM & 0 & 0 & 0 & 0 & 0 & 0 & 0 & 0 & 0 \\
\hline & Present & 0 & 0 & 0 & 0 & 0 & 0 & 0 & 0 & 0 \\
\hline \multirow[t]{3}{*}{$a / 8$} & FEM & 0.0737 & 15.1 & -7.72 & -2.98 & 1.68 & 0.348 & -0.908 & 0.617 & -3.32 \\
\hline & SSM & 0 & 15.1 & -7.68 & -3.00 & 1.66 & 0.353 & -0.902 & 0.609 & -3.23 \\
\hline & Present & 0 & 15.1 & -7.68 & -3.00 & 1.66 & 0.352 & -0.901 & 0.608 & -3.32 \\
\hline \multirow[t]{3}{*}{$a / 4$} & FEM & 0.0668 & 27.1 & -12.6 & -5.41 & 3.43 & 0.853 & -1.08 & 0.787 & -2.75 \\
\hline & SSM & 0 & 27.1 & -12.6 & -5.43 & 3.39 & 0.863 & -1.07 & 0.780 & -2.78 \\
\hline & Present & 0 & 27.1 & -12.6 & -5.43 & 3.40 & 0.863 & -1.06 & 0.780 & -2.75 \\
\hline \multirow[t]{3}{*}{$3 a / 8$} & FEM & 0.0634 & 32.6 & -14.7 & -7.07 & 4.35 & 0.969 & -1.17 & 0.743 & -2.45 \\
\hline & SSM & 0 & 32.6 & -14.5 & -7.09 & 4.30 & 0.983 & -1.16 & 0.735 & -2.46 \\
\hline & Present & 0 & 32.6 & -14.5 & -7.09 & 4.30 & 0.979 & -1.16 & 0.732 & -2.45 \\
\hline \multirow[t]{3}{*}{$a / 2$} & FEM & 0.0632 & 32.4 & -14.5 & -6.98 & 4.29 & 0.898 & -1.08 & 0.676 & -2.21 \\
\hline & SSM & 0 & 32.4 & -14.4 & -7.00 & 4.25 & 0.911 & -1.07 & 0.668 & -2.17 \\
\hline & Present & 0 & 32.4 & -14.4 & -7.00 & 4.25 & 0.913 & -1.06 & 0.670 & -2.20 \\
\hline \multirow[t]{3}{*}{$5 a / 8$} & FEM & 0.0657 & 27.0 & -12.6 & -5.10 & 3.29 & 0.679 & -0.835 & 0.612 & -2.01 \\
\hline & SSM & 0 & 27.0 & -12.5 & -5.11 & 3.25 & 0.689 & -0.824 & 0.606 & -2.00 \\
\hline & Present & 0 & 26.9 & -12.5 & -5.11 & 3.25 & 0.683 & -0.827 & 0.600 & -2.01 \\
\hline \multirow[t]{3}{*}{$3 a / 4$} & FEM & 0.0706 & 16.8 & -8.63 & -2.31 & 1.74 & 0.368 & -0.537 & 0.597 & -2.00 \\
\hline & SSM & 0 & 16.8 & -8.57 & -2.32 & 1.72 & 0.373 & -0.531 & 0.593 & -1.98 \\
\hline & Present & 0 & 16.9 & -8.55 & -2.31 & 1.73 & 0.380 & -0.522 & 0.600 & -1.99 \\
\hline \multirow[t]{3}{*}{$7 a / 8$} & FEM & 0.0771 & 2.48 & -2.94 & 0.0353 & 0.127 & -0.231 & -0.457 & 0.426 & -2.64 \\
\hline & SSM & 0 & 2.51 & -2.91 & 0.0305 & 0.122 & -0.229 & -0.455 & 0.425 & -2.64 \\
\hline & Present & 0 & 2.42 & -2.93 & 0.0273 & 0.109 & -0.246 & -0.469 & 0.408 & -2.64 \\
\hline \multirow[t]{3}{*}{$a$} & FEM & 0.0258 & -155 & -46.7 & -6.72 & -21.3 & -29.9 & -28.0 & -30.6 & 0.718 \\
\hline & SSM & 0 & -153 & -46.5 & -7.07 & -21.6 & -30.0 & -28.1 & -30.8 & 0 \\
\hline & Present & 0 & -155 & -47.0 & -7.16 & -21.7 & -30.3 & -28.4 & -31.0 & 0 \\
\hline
\end{tabular}

Table 13

Bending moment solutions, $\widehat{M}_{2}$, of SCCS cylindrical shell panels.

\begin{tabular}{|c|c|c|c|c|c|c|c|c|c|c|}
\hline \multirow[b]{2}{*}{$\alpha$} & \multirow[b]{2}{*}{$\beta$} & \multicolumn{9}{|l|}{$\widehat{M}_{2}$} \\
\hline & & 0 & $b / 8$ & $b / 4$ & $3 b / 8$ & $b / 2$ & $5 b / 8$ & $3 b / 4$ & $7 b / 8$ & $b$ \\
\hline \multirow[t]{3}{*}{0} & FEM & 0.0264 & 0.0796 & 0.0803 & 0.0803 & 0.0803 & 0.0803 & 0.0803 & 0.0803 & 0 \\
\hline & SSM & 0 & 0 & 0 & 0 & 0 & 0 & 0 & 0 & 0 \\
\hline & Present & 0 & 0 & 0 & 0 & 0 & 0 & 0 & 0 & 0 \\
\hline \multirow[t]{3}{*}{$a / 8$} & FEM & 0.202 & 37.6 & -22.5 & -7.04 & 5.91 & 1.12 & -2.13 & 2.06 & -11.0 \\
\hline & SSM & 0 & 37.6 & -22.3 & -7.07 & 5.86 & 1.15 & -2.10 & 2.05 & -11.1 \\
\hline & Present & 0 & 37.6 & -22.3 & -7.07 & 5.86 & 1.15 & -2.10 & 2.05 & -11.1 \\
\hline \multirow[t]{3}{*}{$a / 4$} & FEM & 0.204 & 72.0 & -39.6 & -13.2 & 10.9 & 1.93 & -3.31 & 2.58 & -9.15 \\
\hline & SSM & 0 & 72.1 & -39.4 & -13.3 & 10.8 & 1.97 & -3.27 & 2.56 & -9.07 \\
\hline & Present & 0 & 72.1 & -39.4 & -13.3 & 10.8 & 1.97 & -3.27 & 2.56 & -9.15 \\
\hline \multirow[t]{3}{*}{$3 a / 8$} & FEM & 0.205 & 90.0 & -47.8 & -17.3 & 13.8 & 2.17 & -3.70 & 2.53 & -8.17 \\
\hline & SSM & 0 & 90.0 & -47.4 & -17.4 & 13.7 & 2.23 & -3.65 & 2.51 & -8.13 \\
\hline & Present & 0 & 90.0 & -47.4 & -17.4 & 13.7 & 2.22 & -3.65 & 2.51 & -8.15 \\
\hline \multirow[t]{3}{*}{$a / 2$} & FEM & 0.205 & 90.8 & -47.9 & -17.4 & 13.7 & 2.06 & -3.46 & 2.34 & -7.35 \\
\hline & SSM & 0 & 90.9 & -47.5 & -17.5 & 13.5 & 2.12 & -3.41 & 2.32 & -7.32 \\
\hline & Present & 0 & 90.8 & -47.5 & -17.5 & 13.5 & 2.11 & -3.40 & 2.32 & -7.32 \\
\hline \multirow[t]{3}{*}{$5 a / 8$} & FEM & 0.204 & 76.3 & -41.1 & -13.5 & 10.6 & 1.74 & -2.76 & 2.12 & -6.71 \\
\hline & SSM & 0 & 76.3 & -40.8 & -13.6 & 10.5 & 1.78 & -2.72 & 2.11 & -6.68 \\
\hline & Present & 0 & 76.3 & -40.8 & -13.5 & 10.5 & 1.78 & -2.72 & 2.11 & -6.69 \\
\hline \multirow[t]{3}{*}{$3 a / 4$} & FEM & 0.203 & 49.1 & -27.7 & -7.88 & 6.09 & 1.27 & -1.86 & 1.98 & -6.66 \\
\hline & SSM & 0 & 49.2 & -27.5 & -7.91 & 6.03 & 1.30 & -1.84 & 1.97 & -6.63 \\
\hline & Present & 0 & 49.2 & -27.5 & -7.90 & 6.03 & 1.29 & -1.83 & 1.97 & -6.65 \\
\hline \multirow[t]{3}{*}{$7 a / 8$} & FEM & 0.201 & 16.7 & -10.8 & -3.21 & 1.90 & 0.568 & -0.907 & 1.48 & -8.79 \\
\hline & SSM & 0 & 16.8 & -10.7 & -3.20 & 1.87 & 0.580 & -0.890 & 1.48 & -8.78 \\
\hline & Present & 0 & 16.7 & -10.7 & -3.20 & 1.87 & 0.572 & -0.891 & 1.48 & -8.81 \\
\hline \multirow[t]{3}{*}{$a$} & FEM & 0.194 & -46.8 & -14.1 & -2.01 & -6.41 & -9.00 & -8.43 & -9.23 & 0.713 \\
\hline & SSM & 0 & -46.0 & -14.0 & -2.12 & -6.47 & -9.02 & -8.48 & -9.15 & 0 \\
\hline & Present & 0 & -46.3 & -14.1 & -2.15 & -6.52 & -9.08 & -8.52 & -9.30 & 0 \\
\hline
\end{tabular}




$$
\begin{aligned}
& -\frac{\mu}{R} \lambda_{m} \bar{u}_{\mathrm{cs}}-\frac{\gamma_{n}}{R} \bar{v}_{\mathrm{sc}}+\left[\frac{1}{R^{2}}+\frac{\delta^{2}}{12}\left(\lambda_{m}^{2}+\gamma_{n}^{2}\right)^{2}\right] \bar{w}_{\mathrm{ss}} \\
& -\frac{\delta^{2}}{12}\left\{\left.\lambda_{m} \int_{0}^{b}\left[\frac{\partial^{2} w}{\partial \alpha^{2}} \cos \left(\lambda_{m} \alpha\right)\right]\right|_{0} ^{a} \sin \left(\gamma_{n} \beta\right) \mathrm{d} \beta\right. \\
& \left.+\left.\gamma_{n} \int_{0}^{a}\left[\frac{\partial^{2} w}{\partial \beta^{2}} \cos \left(\gamma_{n} \beta\right)\right]\right|_{0} ^{b} \sin \left(\lambda_{m} \alpha\right) \mathrm{d} \alpha\right\}=\frac{1-\mu^{2}}{E \delta} \bar{q}_{3 \mathrm{ss}}
\end{aligned}
$$

Denote

$$
\begin{array}{rlrl}
I_{n a} & =\left.\int_{0}^{b} \frac{\partial^{2} w}{\partial \alpha^{2}}\right|_{\alpha=a} \sin \left(\gamma_{n} \beta\right) \mathrm{d} \beta, & I_{n 0}=\left.\int_{0}^{b} \frac{\partial^{2} w}{\partial \alpha^{2}}\right|_{\alpha=0} \sin \left(\gamma_{n} \beta\right) \mathrm{d} \beta \\
J_{m b}=\left.\int_{0}^{a} \frac{\partial^{2} w}{\partial \beta^{2}}\right|_{\beta=b} \sin \left(\lambda_{m} \alpha\right) \mathrm{d} \alpha, & J_{m 0}=\left.\int_{0}^{a} \frac{\partial^{2} w}{\partial \beta^{2}}\right|_{\beta=0} \sin \left(\lambda_{m} \alpha\right) \mathrm{d} \alpha \\
K_{n a}=\left.\int_{0}^{b} \frac{\partial u}{\partial \alpha}\right|_{\alpha=a} \sin \left(\gamma_{n} \beta\right) \mathrm{d} \beta, & K_{n 0}=\left.\int_{0}^{b} \frac{\partial u}{\partial \alpha}\right|_{\alpha=0} \sin \left(\gamma_{n} \beta\right) \mathrm{d} \beta \\
L_{m b}=\left.\int_{0}^{a} \frac{\partial v}{\partial \beta}\right|_{\beta=b} \sin \left(\lambda_{m} \alpha\right) \mathrm{d} \alpha, & L_{m 0}=\left.\int_{0}^{a} \frac{\partial v}{\partial \beta}\right|_{\beta=0} \sin \left(\lambda_{m} \alpha\right) \mathrm{d} \alpha
\end{array}
$$

It follows from Eqs. (18)-(20) that

$$
\left\{\begin{array}{l}
\bar{u}_{\mathrm{cs}} \\
\bar{v}_{\mathrm{sc}} \\
\bar{w}_{\mathrm{ss}}
\end{array}\right\}=\mathbf{T}\left\{\begin{array}{l}
(-1)^{m} K_{n a}-K_{n 0}-\frac{1-\mu^{2}}{E \delta} \bar{q}_{1 \mathrm{cs}} \\
(-1)^{n} L_{m b}-L_{m 0}-\frac{1-\mu^{2}}{E \delta} \bar{q}_{2 \mathrm{sc}} \\
\frac{\delta^{2}}{12}\left\{\lambda_{m}\left[(-1)^{m} I_{n a}-I_{n 0}\right]+\gamma_{n}\left[(-1)^{n} J_{m b}-J_{m 0}\right]\right\}+\frac{1-\mu^{2}}{E \delta} \bar{q}_{3 \mathrm{ss}}
\end{array}\right\}
$$

where the symbolic matrix $\mathbf{T}$ is written as

$$
\mathbf{T}=\left[\begin{array}{ccc}
\lambda_{m}^{2}+\frac{1-\mu}{2} \gamma_{n}^{2} & \frac{1+\mu}{2} \lambda_{m} \gamma_{n} & -\frac{\mu}{R} \lambda_{m} \\
\frac{1+\mu}{2} \lambda_{m} \gamma_{n} & \frac{1-\mu}{2} \lambda_{m}^{2}+\gamma_{n}^{2} & -\frac{1}{R} \gamma_{n} \\
-\frac{\mu}{R} \lambda_{m} & -\frac{1}{R} \gamma_{n} & \frac{1}{R^{2}}+\frac{\delta^{2}}{12}\left(\lambda_{m}^{2}+\gamma_{n}^{2}\right)^{2}
\end{array}\right]^{-1}
$$

Equation (22) establishes the relationship between the transforms of the three displacements and eight sets of unknowns as defined by Eq. (21).

From Eq. (13), incorporating the Stokes transformation (Khalili et al., 2005) for differentiation of trigonometric series, we have

$$
\begin{aligned}
& \frac{\partial w}{\partial \alpha}=\frac{2}{a b} \sum_{m=0}^{\infty} \sum_{n=1}^{\infty} \varepsilon_{m}\left\{\left.\int_{0}^{b}\left[w \cos \left(\lambda_{m} \alpha\right)\right]\right|_{0} ^{a} \sin \left(\gamma_{n} \beta\right) \mathrm{d} \beta+\lambda_{m} \bar{w}_{s s}\right\} \cos \left(\lambda_{m} \alpha\right) \sin \left(\gamma_{n} \beta\right) \\
& \frac{\partial w}{\partial \beta}=\frac{2}{a b} \sum_{m=1}^{\infty} \sum_{n=0}^{\infty} \varepsilon_{n}\left\{\left.\int_{0}^{a}\left[w \cos \left(\gamma_{n} \beta\right)\right]\right|_{0} ^{b} \sin \left(\lambda_{m} \alpha\right) \mathrm{d} \alpha+\gamma_{n} \bar{w}_{s s}\right\} \sin \left(\lambda_{m} \alpha\right) \cos \left(\gamma_{n} \beta\right)
\end{aligned}
$$

Noting $\left.w\right|_{\alpha=0, a}=0$ and $\left.w\right|_{\beta=0, b}=0$, substitution of Eqs. (13) and (24) into the remaining eight boundary conditions that have not been employed, i.e., $\left.u\right|_{\alpha=0, a}=0,\left.\quad v\right|_{\beta=0, b}=0, \quad \partial w /\left.\partial \alpha\right|_{\alpha=0, a}=0$, and $\partial w /\left.\partial \beta\right|_{\beta=0, b}=0$, we have

$$
\begin{aligned}
& \left.\frac{2}{a b} \sum_{m=0}^{\infty} \sum_{n=1}^{\infty} \varepsilon_{m} \bar{u}_{c s} \cos \left(\lambda_{m} \alpha\right) \sin \left(\gamma_{n} \beta\right)\right|_{\alpha=0, a}=0 \\
& \left.\frac{2}{a b} \sum_{m=1}^{\infty} \sum_{n=0}^{\infty} \varepsilon_{n} \bar{v}_{s c} \sin \left(\lambda_{m} \alpha\right) \cos \left(\gamma_{n} \beta\right)\right|_{\beta=0, b}=0 \\
& \left.\frac{4}{a b} \sum_{m=1}^{\infty} \sum_{n=1}^{\infty} \lambda_{m} \bar{w}_{s s} \cos \left(\lambda_{m} \alpha\right) \sin \left(\gamma_{n} \beta\right)\right|_{\alpha=0, a}=0 \\
& \left.\frac{4}{a b} \sum_{m=1}^{\infty} \sum_{n=1}^{\infty} \gamma_{n} \bar{w}_{s s} \sin \left(\lambda_{m} \alpha\right) \cos \left(\gamma_{n} \beta\right)\right|_{\beta=0, b}=0
\end{aligned}
$$

Due to the orthogonality of trigonometric functions, we have

$$
\begin{array}{ll}
\sum_{m=0}^{\infty} \varepsilon_{m} \bar{u}_{\mathrm{cs}}=0 & \text { for } n=1,2,3, \cdots \\
\sum_{m=0}^{\infty}(-1)^{m} \varepsilon_{m} \bar{u}_{\mathrm{cs}}=0 & \text { for } n=1,2,3, \cdots \\
\sum_{n=0}^{\infty} \varepsilon_{n} \bar{v}_{\mathrm{sc}}=0 & \text { for } m=1,2,3, \cdots \\
\sum_{n=0}^{\infty}(-1)^{n} \varepsilon_{n} \bar{v}_{\mathrm{sc}}=0 & \text { for } m=1,2,3, \cdots \\
\sum_{m=1}^{\infty} \lambda_{m} \bar{w}_{\mathrm{ss}}=0 & \text { for } n=1,2,3, \cdots \\
\sum_{m=1}^{\infty}(-1)^{m} \lambda_{m} \bar{w}_{\mathrm{ss}}=0 & \text { for } n=1,2,3, \cdots \\
\sum_{n=1}^{\infty} \gamma_{n} \bar{w}_{\mathrm{ss}}=0 & \text { for } m=1,2,3, \cdots \\
\sum_{n=1}^{\infty}(-1)^{n} \gamma_{n} \bar{w}_{\mathrm{ss}}=0 & \text { for } m=1,2,3, \cdots
\end{array}
$$

Substituting Eq. (22) into Eq. (26), we obtain eight infinite systems of linear simultaneous equations with respect to the eight sets of unknowns as defined by Eq. (21). In actual computation, finite series terms should be taken. In other words, instead of $\infty$, the prescribed positive integers, $M$ and $N$, can be taken in Eq. (26) as the upper limits of $m$ and $n$, respectively. Accordingly, we have $4(M+N)$ equations with the same number of solvable constants, i.e., $I_{n 0}, I_{n a}, K_{n 0}, K_{n a}(n=1,2,3, \cdots, N)$ and $J_{m 0}, J_{m b}, L_{m 0}, L_{m b}(m=1,2,3, \cdots, M)$. Substituting the determined constants into Eq. (22) then into Eq. (13) leads to the analytical displacement solutions of CCCC cylindrical shell panels. Any other mechanical quantities can be further obtained. For instance, the internal forces can be readily pursued by Eqs. (4)-(11).

\section{Cylindrical shell panels with other combinations of clamped and simply supported edges}

To further demonstrate the generality of the present double finite integral transform method, we explore the analytical solutions of nonLévy-type cylindrical shell panels with other combinations of clamped and simply supported edges.

For a CCCS panel, the edge at $\alpha=0$ is simply supported and the other three edges are clamped; thus, the boundary conditions are written as

$$
\begin{aligned}
& \left.\left(F_{T 1}, v, w, M_{1}\right)\right|_{\alpha=0}=0 \\
& \left.\left(u, v, w, \frac{\partial w}{\partial \alpha}\right)\right|_{\alpha=a}=0 \\
& \left.\left(u, v, w, \frac{\partial w}{\partial \beta}\right)\right|_{\beta=0}=0 \\
& \left.\left(u, v, w, \frac{\partial w}{\partial \beta}\right)\right|_{\beta=b}=0
\end{aligned}
$$

Compared with the derivation of the CCCC panel, the new conditions 


$$
\begin{aligned}
& \left.F_{T 1}\right|_{\alpha=0}=\left.\frac{E \delta}{1-\mu^{2}}\left[\frac{\partial u}{\partial \alpha}+\mu\left(\frac{\partial v}{\partial \beta}+\frac{w}{R}\right)\right]\right|_{\alpha=0}=0 \\
& \left.M_{1}\right|_{\alpha=0}=-\left.\frac{E \delta^{3}}{12\left(1-\mu^{2}\right)}\left(\frac{\partial^{2} w}{\partial \alpha^{2}}+\mu \frac{\partial^{2} w}{\partial \beta^{2}}\right)\right|_{\alpha=0}=0
\end{aligned}
$$

yield

$$
\begin{aligned}
& I_{n 0}=\left.\int_{0}^{b} \frac{\partial^{2} w}{\partial \alpha^{2}}\right|_{\alpha=0} \sin \left(\gamma_{n} \beta\right) \mathrm{d} \beta=0 \\
& K_{n 0}=\left.\int_{0}^{b} \frac{\partial u}{\partial \alpha}\right|_{\alpha=0} \sin \left(\gamma_{n} \beta\right) \mathrm{d} \beta=\left.\int_{0}^{b}\left(-\mu \frac{\partial v}{\partial \beta}\right)\right|_{\alpha=0} \\
& \sin \left(\gamma_{n} \beta\right) \mathrm{d} \beta=-\left.\mu\left[\left.\sin \left(\gamma_{n} \beta\right) v\right|_{\alpha=0}\right]\right|_{0} ^{b}+\left.\mu \gamma_{n} \int_{0}^{b} v\right|_{\alpha=0} \cos \left(\gamma_{n} \beta\right) \mathrm{d} \beta=0
\end{aligned}
$$

Therefore, a simplification of Eq. (22) gives

$$
\left\{\begin{array}{l}
\bar{u}_{\mathrm{cs}} \\
\bar{v}_{\mathrm{sc}} \\
\bar{w}_{\mathrm{ss}}
\end{array}\right\}=\mathbf{T}\left\{\begin{array}{l}
(-1)^{m} K_{n a}-\frac{1-\mu^{2}}{E \delta} \bar{q}_{1 \mathrm{cs}} \\
(-1)^{n} L_{m b}-L_{m 0}-\frac{1-\mu^{2}}{E \delta} \bar{q}_{2 \mathrm{sc}} \\
\frac{\delta^{2}}{12}\left\{(-1)^{m} \lambda_{m} I_{n a}+\gamma_{n}\left[(-1)^{n} J_{m b}-J_{m 0}\right]\right\}+\frac{1-\mu^{2}}{E \delta} \bar{q}_{3 \mathrm{ss}}
\end{array}\right\}
$$

Following the same logic as depicted in section 2.3, satisfying the remaining six boundary conditions yields six infinite systems of linear simultaneous equations with respect to the six sets of unknowns. Substituting the determined constants into Eq. (30) then into Eq. (13) leads to the analytical solutions of CCCS panels. The solutions for the other cases can be obtained with the same procedure. For the sake of brevity, only the boundary conditions and the relationships between the transforms of the three displacements and the unknowns are presented.

For a SCCC panel, the boundary conditions are

$$
\begin{aligned}
& \left.\left(u, v, w, \frac{\partial w}{\partial \alpha}\right)\right|_{\alpha=0}=0 \\
& \left.\left(u, v, w, \frac{\partial w}{\partial \alpha}\right)\right|_{\alpha=a}=0 \\
& \left.\left(F_{T 2}, v, w, M_{2}\right)\right|_{\beta=0}=0 \\
& \left.\left(u, v, w, \frac{\partial w}{\partial \beta}\right)\right|_{\beta=b}=0
\end{aligned}
$$

The corresponding relationship is

$$
\left\{\begin{array}{l}
\bar{u}_{\mathrm{cs}} \\
\bar{v}_{\mathrm{sc}} \\
\bar{w}_{\mathrm{ss}}
\end{array}\right\}=\mathbf{T}\left\{\begin{array}{l}
(-1)^{m} K_{n a}-K_{n 0}-\frac{1-\mu^{2}}{E \delta} \bar{q}_{1 \mathrm{cs}} \\
(-1)^{n} L_{m b}-\frac{1-\mu^{2}}{E \delta} \bar{q}_{2 \mathrm{sc}} \\
\frac{\delta^{2}}{12}\left\{\lambda_{m}\left[(-1)^{m} I_{n a}-I_{n 0}\right]+(-1)^{n} \gamma_{n} J_{m b}\right\}+\frac{1-\mu^{2}}{E \delta} \bar{q}_{3 \mathrm{ss}}
\end{array}\right\}
$$

For a SCCS panel, the boundary conditions are

$$
\begin{aligned}
& \left.\left(F_{T 1}, v, w, M_{1}\right)\right|_{\alpha=0}=0 \\
& \left.\left(u, v, w, \frac{\partial w}{\partial \alpha}\right)\right|_{\alpha=a}=0 \\
& \left.\left(F_{T 2}, v, w, M_{2}\right)\right|_{\beta=0}=0 \\
& \left.\left(u, v, w, \frac{\partial w}{\partial \beta}\right)\right|_{\beta=b}=0
\end{aligned}
$$

The corresponding relationship is

$$
\left\{\begin{array}{l}
\bar{u}_{\mathrm{cs}} \\
\bar{v}_{\mathrm{sc}} \\
\bar{w}_{\mathrm{ss}}
\end{array}\right\}=\mathbf{T}\left\{\begin{array}{l}
(-1)^{m} K_{n a}-\frac{1-\mu^{2}}{E \delta} \bar{q}_{1 \mathrm{cs}} \\
(-1)^{n} L_{m b}-\frac{1-\mu^{2}}{E \delta} \bar{q}_{2 \mathrm{sc}} \\
\frac{\delta^{2}}{12}\left[(-1)^{m} \lambda_{m} I_{n a}+(-1)^{n} \gamma_{n} J_{m b}\right]+\frac{1-\mu^{2}}{E \delta} \bar{q}_{3 \mathrm{ss}}
\end{array}\right\}
$$

\section{Comprehensive numerical results with validations}

Comprehensive numerical results of CCCC, SCCC, CCCS, and SCCS cylindrical shell panels with $R / a=1, b / a=1$ and uniform radial loading of intensity $q$ are given to reveal the validity of this study. The nondimensional displacement solutions, $\widehat{u}=10^{7} u E \delta^{3} /\left(q a^{4}\right), \quad \widehat{v}=$ $10^{7} v E \delta^{3} /\left(q a^{4}\right)$, and $\widehat{w}=10^{7} w E \delta^{3} /\left(q a^{4}\right)$, and non-dimensional bending moments, $\widehat{M}_{1}=10^{5} M_{1} /\left(q a^{2}\right)$ and $\widehat{M}_{2}=10^{5} M_{2} /\left(q a^{2}\right)$, are adopted.

The convergence study of CCCC panels is first carried out for the displacements and bending moments at typical locations, as shown in Tables 1 and 2, respectively, where the bold figures are the convergent ones. Overall, with the increase of the number of series terms, the displacement solutions converge fast whereas the bending moments converge slower since they are obtained by the second derivatives of $w$. Eventually, 400 series terms have been taken throughout to achieve the accuracy of four significant digits for all the displacement solutions, and 2000 series terms achieve the accuracy of three significant digits for all the bending moment solutions in this study. The FEM-based numerical solutions have been obtained by the ABAQUS software (ABAQUS, 2013) for comparison, where the Poisson's ratio and thickness-to-span ratio are set to be 0.3 and $10^{-3}$, respectively. The adopted element is C3D20R solid element. A fine enough mesh is set, with the mesh size being $1 / 200$ of $a$, which outputs numerical solutions with high accuracy. The results by an up-to-date symplectic superposition method (SSM) have also been presented for comparison (Zheng et al., 2019). Satisfactory agreement is found between the present analytical solutions and those by FEM as well as SSM, confirming the accuracy of the analytical results.

Comprehensive non-dimensional displacement solutions of CCCC, CCCS, and SCCC panels are tabulated in Tables $3-5$, respectively. $\widehat{u}, \widehat{v}$, and $\widehat{w}$ of SCCS panels are tabulated in Tables $6-8$, respectively. The nondimensional bending moments of CCCC, CCCS, and SCCC panels are tabulated in Tables 9-11, respectively. Tables 12 and 13 present $\widehat{M}_{1}$ and $\widehat{M}_{2}$ of SCCS panels. Overall, 408 displacements and 392 bending moments at different locations have been listed to provide comprehensive benchmark numerical results. All the present analytical solutions agree well with the reference ones. Compared with the analytical SSM that involves unusual mathematical skills (Zheng et al., 2019), the present finite integral transform method is more straightforward, which provides an easy-to-implement tool for analytical modeling of similar mechanics problems.

\section{Concluding remarks}

This study demonstrates the analytical double finite integral transform method for static bending problems of cylindrical shell panels. The theoretical foundation of the method is solid and the solution procedure is succinct without introducing any abstruse mathematical skills, which qualifies the method as a promising straightforward tool that may attract more extensive applications than some other analytical methods. In view of the merits on dealing with complex boundary-value problems of high-order PDEs, the follow-up studies on the method may be oriented towards more difficult issues such as bending, vibration, and buckling of intricate shell structures.

It is worth pointing out that the double finite integral transform method is also applicable to the panels with free edge(s), although this study focuses on those without a free edge. However, different integral transform kernels may be adopted. In general, taking the displacement $w$ 
as an example, one could follow the guidelines below to choose the kernels provided that a similar coordinate system to this study is taken. For a pair of opposite edges, a sinusoidal kernel is adopted in the corresponding direction when the both edges are clamped or simply supported, or one of them is clamped and another simply supported; a halfsinusoidal/cosinusoidal kernel is adopted when one of them is clamped or simply supported and another free; a cosinusoidal kernel is adopted when the both edges are free. The panels in this study fall into the first category, thus the sinusoidal kernels are adopted in both directions for $w$.

\section{Declaration of competing interests}

The authors declare that they have no known competing financial interests or personal relationships that could have appeared to influence the work reported in this paper.

\section{Acknowledgements}

The authors gratefully acknowledge the support from the National Natural Science Foundation of China (Grant Nos. 11972103 and 11825202) and Liaoning Revitalization Talents Program (Grant Nos. XLYC1807126 and XLYC1802020). Y.S. gratefully acknowledges the support from Beijing Municipal Science and Technology Commission (Z191100002019010), Beijing Municipal Natural Science Foundation (No. 2202066), Key Research Program of Frontier Sciences of the Chinese Academy of Sciences (ZDBS-LY-JSC014), Strategic Priority Research Program of the Chinese Academy of Sciences (No. XDB22040501), and State Key Laboratory of Structural Analysis for Industrial Equipment, Dalian University of Technology (No. GZ19102).

\section{References}

ABAQUS, 2013. Analysis User's Guide V6.13. Dassault Systèmes, Pawtucket, RI.

Achryya, A.K., Chakravorty, D., Karmakar, A., 2009. Bending characteristics of delaminated composite cylindrical shells - a finite element approach. J. Reinforc. Plast. Compos. 28, 965-978.

An, C., Su, J., 2011. Dynamic response of clamped axially moving beams: integral transform solution. Appl. Math. Comput. 218, 249-259.

Assaee, H., Hasani, H., 2015. Forced vibration analysis of composite cylindrical shells using spline finite strip method. Thin-Walled Struct. 97, 207-214.

Chen, S.W., Liu, H.M., Peng, Y., Sun, J.L., 2013. Strip layer method for simulation of the three-dimensional deformations of large cylindrical shell rolling. Int. J. Mech. Sci. 77, 113-120.

Cinefra, M., Belouettar, S., Soave, M., Carrera, E., 2010. Variable kinematic models applied to free-vibration analysis of functionally graded material shells. Eur. J. Mech. A Solid. 29, 1078-1087.

Cotta, R.M., Knupp, D.C., Naveira-Cotta, C.P., Sphaier, L.A., Quaresma, J.N.N., 2013. Unified integral transforms algorithm for solving multidimensional nonlinear convection-diffusion problems. Numer. Heat Tr.A-Appl. 63, 840-866.

Dirgantara, T., Aliabadi, M.H., 2012. Elastoplastic boundary element method for shear deformable shells. Eng. Struct. 45, 62-67.
Ferreira, A.J.M., Carrera, E., Cinefra, M., Roque, C.M.C., 2011. Analysis of laminated doubly-curved shells by a layerwise theory and radial basis functions collocation, accounting for through-the-thickness deformations. Comput. Mech. 48, 13-25.

He, Y., An, C., Su, J., 2020a. Generalized integral transform solution for free vibration of orthotropic rectangular plates with free edges. J. Brazilian Soc. Mech. Sci. Eng. 42, 183.

He, Y., An, C., Su, J., 2020b. Bending of orthotropic rectangular thin plates with two opposite edges clamped. Proc. Inst. Mech. Eng. Part C J. Mech. Eng. Sci. 234, $1220-1230$.

Khalili, M.R., Malekzadeh, K., Mittal, R.K., 2005. A new approach to static and dynamic analysis of composite plates with different boundary conditions. Compos. Struct. 69, $149-155$.

Leissa, A.W., 1973. Vibration of Shells. Scientific and Technical Information Office, National Aeronautics and Space Administration., Washington, D.C.

Li, H., Pang, F., Chen, H., 2019. A semi-analytical approach to analyze vibration characteristics of uniform and stepped annular-spherical shells with general boundary conditions. Eur. J. Mech. A Solid. 74, 48-65.

Li, R., Wang, H., Zheng, X., Xiong, S., Hu, Z., Yan, X., Xiao, Z., Xu, H., Li, P., 2019. New analytic buckling solutions of rectangular thin plates with two free adjacent edges by the symplectic superposition method. Eur. J. Mech. A Solid. 76, 247-262.

Li, R., Zhong, Y., Tian, B., Liu, Y., 2009. On the finite integral transform method for exact bending solutions of fully clamped orthotropic rectangular thin plates. Appl. Math. Lett. 22, 1821-1827.

Lim, C.W., Xu, X.S., 2010. Symplectic elasticity: theory and applications. Appl. Mech. Rev. 63, 050802.

Lim, C.W., Lü, C.F., Xiang, Y., Yao, W., 2009. On new symplectic elasticity approach for exact free vibration solutions of rectangular Kirchhoff plates. Int. J. Eng. Sci. 47, 131-140.

Matt, C.F.T., 2013. Combined classical and generalized integral transform approaches for the analysis of the dynamic behavior of a damaged structure. Appl. Math. Model. 37, 8431-8450.

Mousavi, S.M., Aghdam, M.M., 2009. Static bending analysis of laminated cylindrical panels with various boundary conditions using the differential cubature method. J. Mech. Mater. Struct. 4, 509-521.

Ortakaya, S., 2012. Exact solutions of the Klein - gordon equation with ring-shaped oscillator potential by using the Laplace integral transform. Chin. Phys. B 21, 070303.

Santos, E.N., Blanco, C.J.C., MacÊdo, E.N., Maneschy, C.E.A., Quaresma, J.N.N., 2012. Integral transform solutions for the analysis of hydrodynamic lubrication of journal bearings. Tribol. Int. 52, 161-169.

Silva, R.L., Quaresma, J.N.N., Santos, C.A.C., Cotta, R.M., 2011. Integral transforms solution for flow development in wavy wall ducts. Int. J. Numer. Method. H. 21, 219-243.

Sneddon, I.N., 1975. Application of Integral Transforms in the Theory of Elasticity. McGraw-Hill, New York.

Timoshenko, S., Woinowsky-Krieger, S., 1959. Theory of Plates and Shells. McGraw-Hill, Auckland.

Ullah, S., Wang, H., Zheng, X., Zhang, J., Zhong, Y., Li, R., 2019. New analytic buckling solutions of moderately thick clamped rectangular plates by a straightforward finite integral transform method. Arch. Appl. Mech. 89, 1885-1897.

Yang, X.J., 2016. A new integral transform method for solving steady heat-transfer problem. Therm. Sci. 20, S639-S642.

Zhang, J., Ullah, S., Zhong, Y., 2020. New analytical free vibration solutions of orthotropic rectangular thin plates using generalized integral transformation. J. Comput. Appl. Math. 367, 112439.

Zhang, S., Xu, L., Li, R., 2019. New exact series solutions for transverse vibration of rotationally-restrained orthotropic plates. Appl. Math. Model. 65, 348-360.

Zhang, X., Cai, J., Liu, L., Yang, Y., 2012. An integral transform and its applications in parameter estimation of LFM signals. Circ. Syst. Signal Process. 31, 1017-1031.

Zheng, X., Sun, Y., Huang, M., An, D., Li, P., Wang, B., Li, R., 2019. Symplectic superposition method-based new analytic bending solutions of cylindrical shell panels. Int. J. Mech. Sci. 152, 432-442. 